\title{
Mineral InCLUSIONS IN SAPPHIRE FROM BASALTIC TERRANES IN SOUTHERN VietNAM: INDICATOR OF FORMATION MODEL
}

Doan Thi Anh Vu, Abhisit Salam, Alongkot Fanka, Elena Belousova, and Chakkaphan Sutthirat

Sapphires in southern Vietnam have been discovered in alluvial gem fields derived from Cenozoic basalts. Several syngenetic mineral inclusions were identified by Raman spectroscopy and electron probe microanalysis; they were classified as ferrocolumbite, zircon, alkali feldspar (albite-anorthoclase-oligoclase), pyrochlore, hercynite spinel, and ilmenite (titanohematite series). Geochemical characteristics of these inclusions appear to have evolved from an alkaline felsic suite. Consequently, the original formation of these sapphires would be related to alkaline felsic magmatic processes before basaltic magmatism, which was derived from the deeper upper mantle, had passed through and transported the sapphires and their associated minerals to the surface via volcanic eruption.

S ince the late 1980s, sapphire mines in southern Vietnam have supplied significant quantities of gem materials to the jewelry industry (figure 1). Commercial gem mines in southern Vietnam have been located in four main areas, including Dak Nong, Di Linh, Binh Thuan, and Krong Nang (figure 2) (Smith et al., 1995; Long et al., 2004; Garnier et al., 2005; Izokh et al., 2010; Vu, 2010, 2018). Specifically, the first discovery of abundant gem sapphires was in Dak Nong, followed by Di Linh, Binh Thuan, and Krong Nang, respectively. These sapphires accumulated in Upper-Pleistocene to Quaternary alluvial deposits.

In 2017, we carried out field trips in southern Vietnam encompassing Binh Thuan Province, Di Linh (Lam Dong Province), Dak Nong Province, and Krong Nang (Dak Lak Province), where sapphire samples were collected directly from the mines (see figure 2). Gem mining activities in these areas are conducted by a few local miners. Pits are dug with basic tools before washing and hand picking along streams by artisanal miners (figure 3), and mines worked by machinery (figure 4) can also be found. Sapphires in this region usually range from dark blue to bluish green, yellowish green to green, with rare yellow sapphire. The natural intense blue sapphire is

See end of article for About the Authors and Acknowledgments.

Gems \& Gemology, Vol. 56, No. 4, pp. 498-515,

http://dx.doi.org/10.5741/GEMS.56.4.498

(C) 2020 Gemological Institute of America the best known and has been specifically recovered from the Dak Nong and Di Linh gem fields (figure 1, left). Other colors such as bluish green and yellowish green to green are common, particularly in the Binh Thuan and Krong Nang gem fields, respectively (figure 1, right). However, these sapphire varieties are generally heat treated for color enhancement. In addition, trapiche-type sapphires (figure 5) are sometimes found in these gem fields.

\section{In Brief}

- Several syngenetic mineral inclusions were recognized in sapphires from southern Vietnam.

- Chemical compositions of these mineral inclusions indicated that they evolved from an alkaline felsic suite.

- Alkaline felsic melts were then proposed as the original formation environment of sapphire from basaltic terranes in southern Vietnam.

Gemological properties and chemical fingerprints of sapphire from southern Vietnam have identified this material as basaltic-type (Smith et al., 1995; Long et al., 2004; Garnier et al., 2005; Izokh et al., 2010; $\mathrm{Vu}, 2010,2018)$. However, understanding of their formation remains unresolved. Inclusions in sapphire, including basaltic type, have become a highly interesting issue in the last decade. These inclusions may preserve significant information on chemical and 

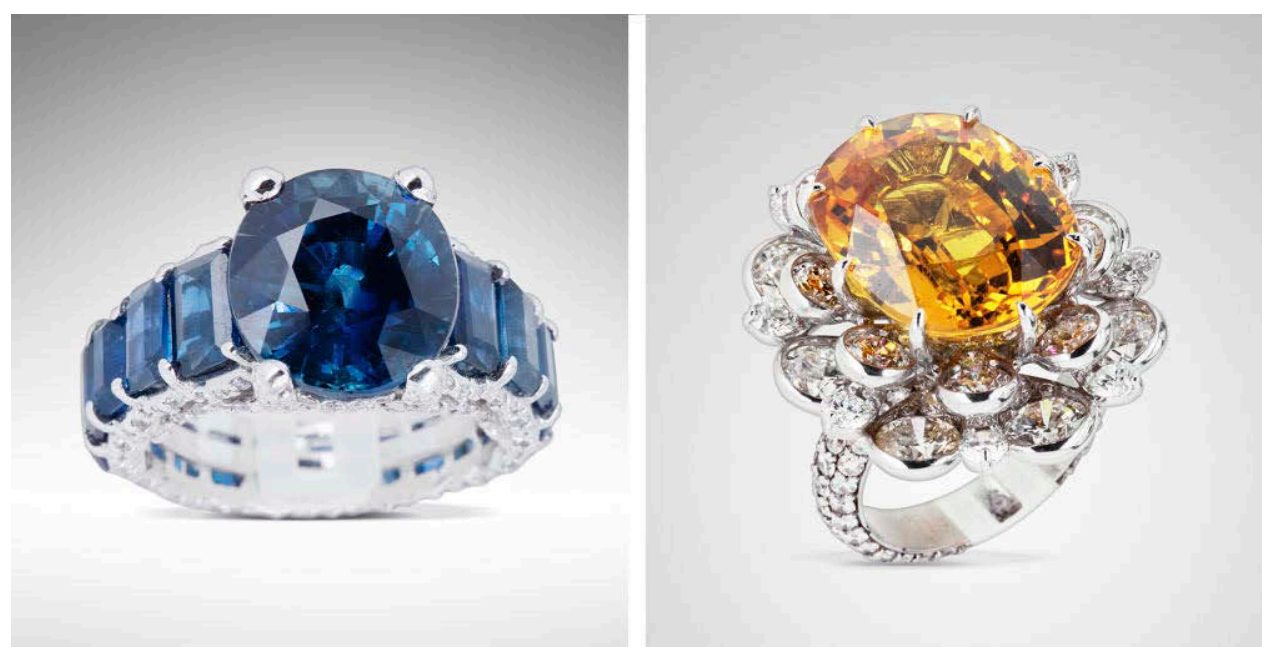

Figure 1. High-quality sapphires from southern Vietnam set in rings. Left: The center blue sapphire is approximately $8.8 \mathrm{ct}$, and the side stones range from 0.3 to $0.4 \mathrm{ct}$; all are from Di Linh. Right: An approximately $18 \mathrm{ct}$ yellow sapphire from Binh Thuan surrounded by small diamonds. The yellow sapphire was heated to improve its color. Photos by Doan Thi Anh Vu.

physical conditions directly related to the original

quently, various analytical techniques such as formation of the basaltic-type sapphire. Subse-

Raman spectroscopy, scanning electron microscopy

Figure 2. Map of Vietnam showing the distribution of Cenozoic basalts and the main sapphire mining areas in southern Vietnam with the four occurrences where samples were collected (Binh Thuan, Di Linh, Dak Nong, and Krong Nang); modified from Hoa et al. (2005), Lepvrier et al. (2008), Tri and Khuc (2011), and Hoang et al. (2018).
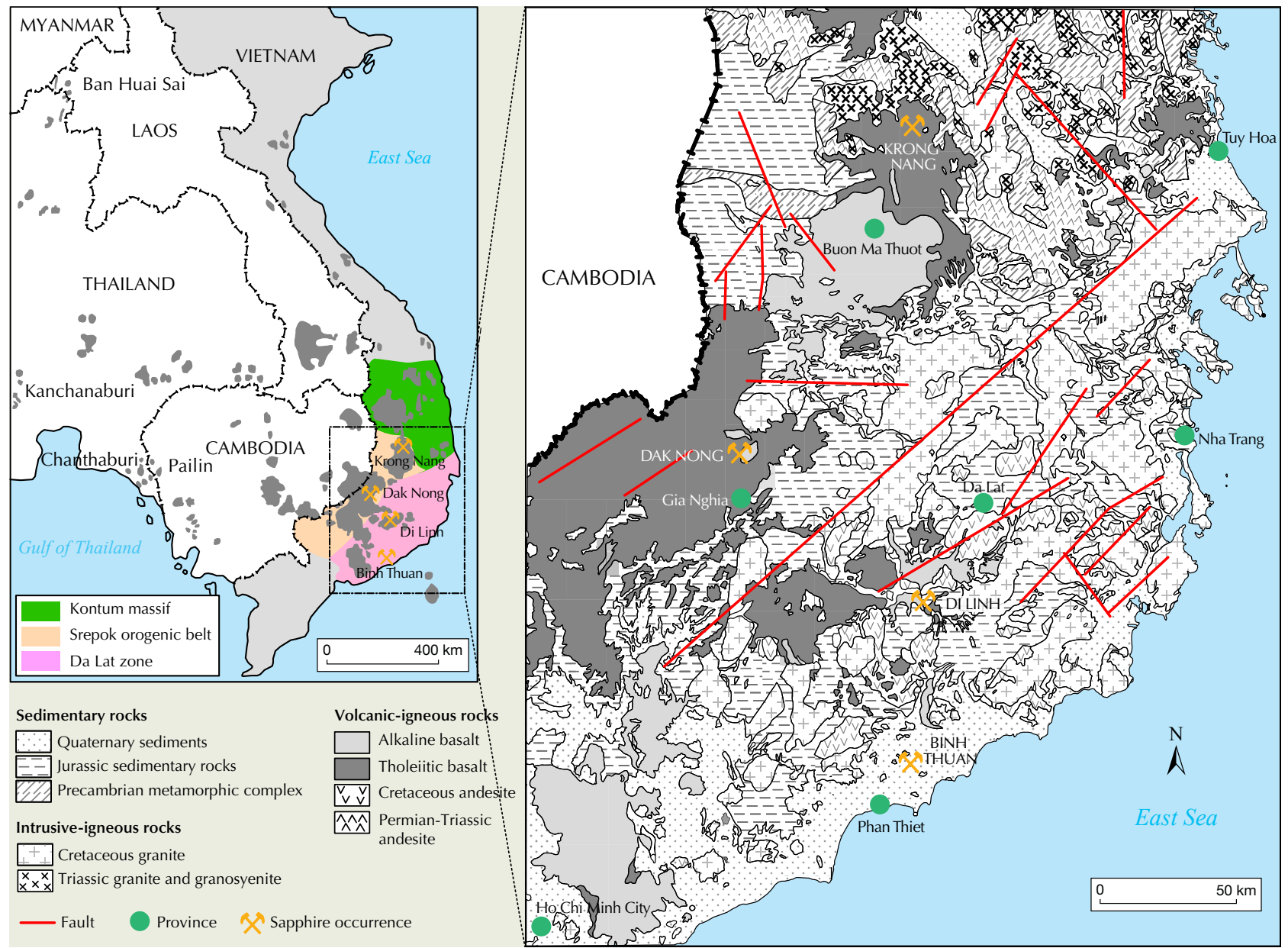

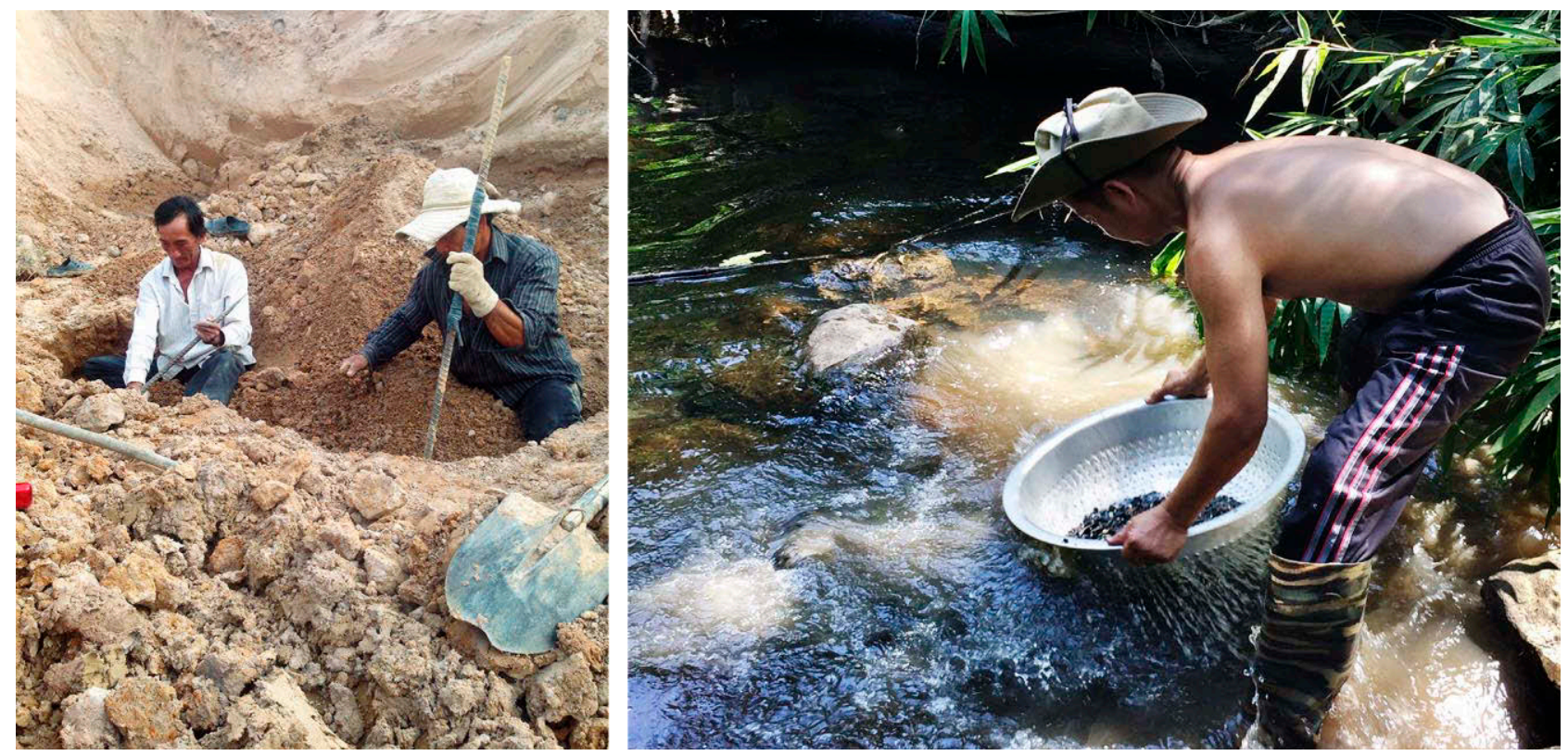

Figure 3. Left: Artisanal miners use crowbars and shovels to remove topsoil and dig through the gem-bearing layer in the Binh Thuan gem field. Right: Gem-bearing gravels are washed and sieved along the stream at Dak Nong. Photos by Doan Thi Anh Vu.

with energy-dispersive spectroscopy (SEM-EDS), and electron probe microanalysis (EPMA) have been applied for identification of inclusions in sapphire from southern Vietnam. For example, Smith et al. (1995) and Long et al. (2004) used SEM-EDS to identify various mineral inclusions in sapphires from Dak Nong and Phan Thiet, whereas Izokh et al. (2010) applied
EPMA for chemical analyses of a few inclusions in sapphires from Dak Nong.

Although a variety of mineral inclusions in sapphires from southern Vietnam were previously reported, the chemistry of these inclusions has not been widely analyzed. Therefore, this study is focused on a wider variety of mineral inclusions and

Figure 4. In areas mined with machinery, backhoes were used to remove topsoil and reach the gem-bearing gravel layers prior to washing and sieving using a water pump in the gem fields of Binh Thuan (left) and Krong Nang (right). Photos by Doan Thi Anh Vu.
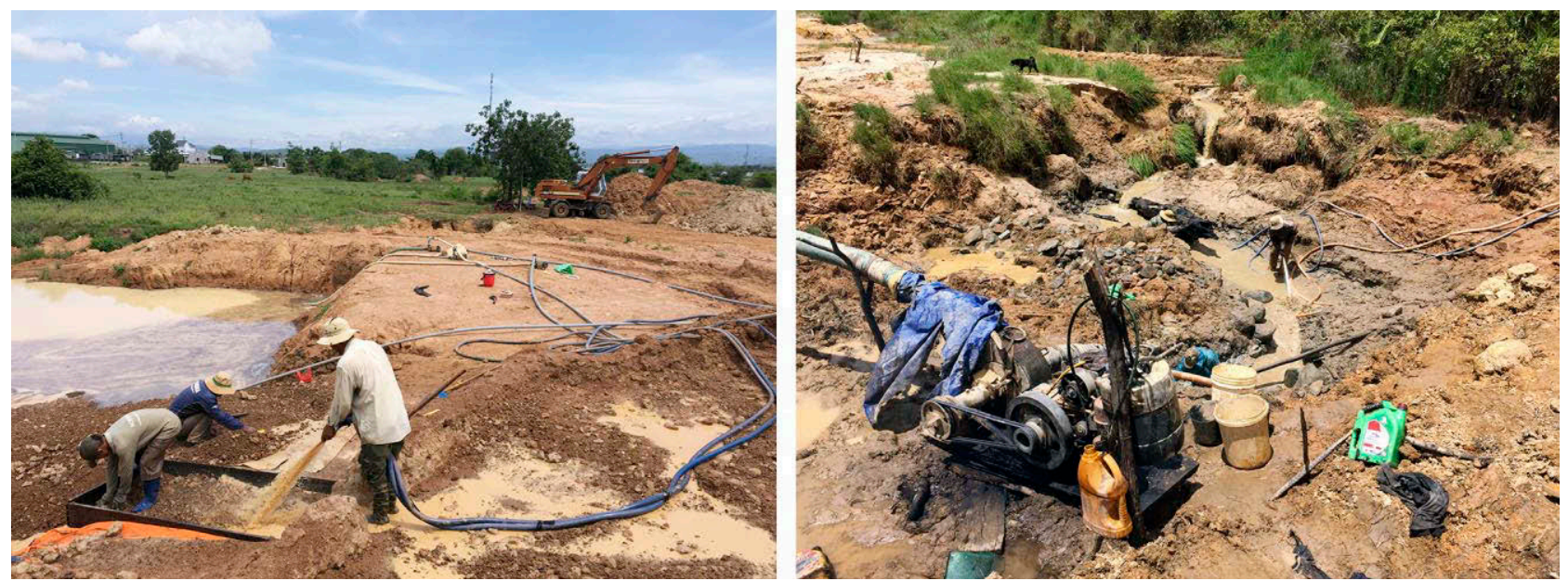


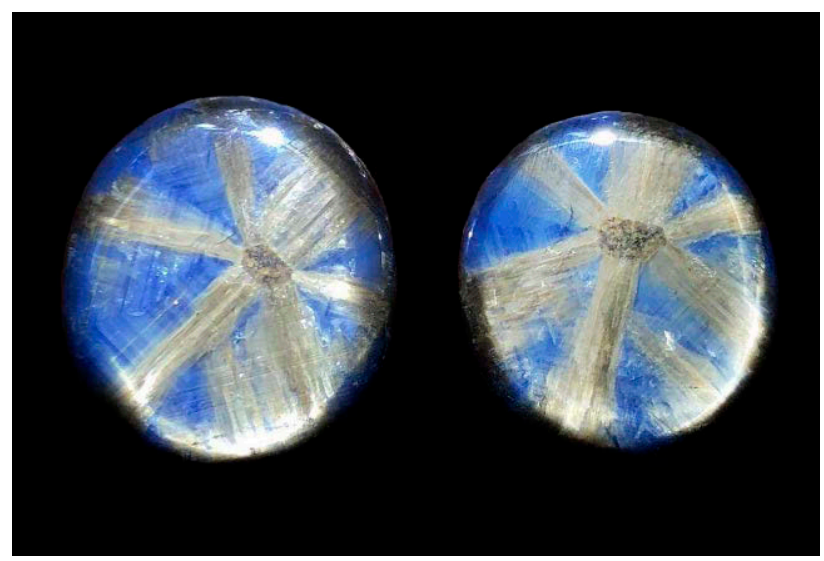

Figure 5. Trapiche-type sapphires, about $10 \mathrm{ct}$ (left) and 9 ct (right), from Binh Thuan gem field. Photo by Doan Thi Anh Vu.

their chemical analyses using EPMA. These analytical data lead to a reconstruction of a genetic model of sapphire formation related to basaltic volcanism in this region. They also yield significant information for origin determination by gem testing laboratories.

\section{GEOLOGICAL SETTING}

Southern Vietnam geologically belongs to a largescale structure of the Da Lat active continental margin (Da Lat zone) and the Indosinian polyepisodic orogenic belt (Srepok orogenic belt) (figure 2, left) (Tri and Khuc, 2011), which separated from Gondwana in the Devonian (Şengör et al., 1988; Hutchison, 1989; Metcalfe, 1988, 1996, 2009, 2011). Rock formations range widely: Precambrian basement rocks, Jurassic sedimentary rocks, late Mesozoic igneous rocks, and Cenozoic basaltic rocks (Hoa et al., 2005) (figure 2, right). The basement rocks in this area are composed of metamorphic complexes of granulites and gneiss granulite. A thick pile of Jurassic rocks covering the basement rocks is composed of sandstone, siltstone, and shale. These basement rocks and Jurassic rocks are intruded by a number of late Mesozoic rocks, including Triassic granite and granosyenite and Cretaceous granite.

The uppermost part of the area was eventually covered by Cenozoic basalts, which are directly associated with sapphires in southern Vietnam. These Cenozoic (Neogene-Quaternary) basalts range in age from 0.8 to 17.6 Ma (Hoang and Flower, 1998; Garnier et al., 2005) and appear to have been related to the most recent tectonic event, the opening of the East Sea after the Indian-Eurasian collision (from 35 to $17 \mathrm{Ma}$ ) (Barr and MacDonald, 1981; Rangin et al., 1995; Hoang and Flower, 1998; Lee et al., 1998). Ac- cording to Hoang and Flower (1998), these basalts covering an area of about $23,000 \mathrm{~km}^{2}$ with a thickness of several hundred meters erupted in two main episodes. The early stages mostly included tholeiite basalts, which erupted from extensional fissures trending along the NE-SW and NW-SE directions, derived from the lithosphere. On the other hand, the latter alkali basalts usually flowed along conjugate strike-slip faults originating from the asthenosphere. Only the alkali basalts in particular are associated with sapphire (Smith et al., 1995; Garnier et al., 2005; Izokh et al., 2010). According to Garnier et al. (2005), alkali basalts in Dak Nong are characterized by porphyritic olivine basalts that comprise olivine, clinopyroxene, plagioclase, and spinel. These compositions are associated with megacrysts, xenocrysts, and xenoliths of the mantle and lower crust. Mantle xenoliths embedded in Dak Nong basalts are recognized as garnet lherzolite and spinel lherzolite, while xenocrysts include olivine, Al-rich clinopyroxene, orthopyroxene, phlogopite, sapphire, and zircon. Lower crustal xenoliths usually contain plagioclase and quartz. The presence of mantle xenoliths, xenocrysts, and lower crustal xenoliths may indicate that crystallization of these minerals occurred at high pressure in a magma chamber close to the boundary between the upper mantle and lower crust.

\section{MATERIALS AND METHODS}

Over a thousand unheated, gem-quality rough sapphires were collected directly from the gem fields in southern Vietnam (Dak Nong, Di Linh, Krong Nang, and Binh Thuan) for this study. Most of the samples from the Dak Nong and Di Linh mines were collected by the first author over 20 years, while sapphires from Binh Thuan and Krong Nang were collected in 2001 and 2012, respectively. Moreover, more samples from all areas were collected by the authors during the field trip in 2017. These were initially investigated under a gemological microscope to find suitable samples containing mineral inclusions. Subsequently, a total of 274 sapphire samples ( 77 from Dak Nong, 75 from Di Linh, 45 from Krong Nang, and 77 from Binh Thuan) were then selected and polished to expose the inclusions.

These inclusions were then identified by a Renishaw InVia 1000 laser Raman spectroscope at the Gem and Jewellery Institute of Thailand (Public Organization) (GIT). Subsequently, they were analyzed for major and minor compositions using a JEOL JXA8100 electron probe microanalyzer at the Depart- 
ment of Geology, Faculty of Science, Chulalongkorn University. Operating conditions were set at $15 \mathrm{kV}$ acceleration voltage and $24 \mathrm{nA}$ filament current with 30 seconds of peak and background counts for each element prior to automatic ZAF calculation and reporting in oxide forms. The general detection limits of major and minor analyses obtained from EPMA are typically lower than 0.01 oxide wt. \%. Various natural minerals and artificial standards were used for calibration. These included quartz, corundum, eskolaite, fayalite, manganosite, periclase, nickel oxide, wollastonite, jadeite, potassium titanium phosphate, strontium barium niobate, zirconium, yttrium phosphate, cerium phosphate, and neodymium phosphate for $\mathrm{SiO}_{2}, \mathrm{Al}_{2} \mathrm{O}_{3}, \mathrm{Cr}_{2} \mathrm{O}_{3}, \mathrm{FeO}, \mathrm{MnO}, \mathrm{MgO}, \mathrm{NiO}, \mathrm{CaO}$, $\mathrm{Na}_{2} \mathrm{O}, \mathrm{K}_{2} \mathrm{O}, \mathrm{Nb}_{2} \mathrm{O}_{5}, \mathrm{ZrO}_{2}, \mathrm{Y}_{2} \mathrm{O}_{3}, \mathrm{Ce}_{2} \mathrm{O}_{3}$, and $\mathrm{Nd}_{2} \mathrm{O}_{3^{\prime}}$ respectively. $\mathrm{Fe}^{2+}$ and $\mathrm{Fe}^{3+}$ ratios of some specific minerals (e.g., spinel and ilmenite) were estimated by stoichiometric calculation as suggested by Droop (1987). Additionally, a total of four samples that contained zircon inclusions, one from each area, were analyzed for their rare earth elements (REE). These were analyzed using an Agilent 7700 quadrupole ICP-MS instrument, attached to a Photon Machines Excimer $193 \mathrm{~nm}$ laser ablation (LA) system at Macquarie University in Sydney, Australia. The analyses were carried out using the same laser conditions as for U-Pb dating. Detailed descriptions of analytical and calibration procedures were given by Belousova et al. (2002). These zircon inclusions were analyzed using a $2 \mathrm{~Hz}$ pulse rate with about $0.5 \mathrm{~m}$ J beam energy per pulse, achieving a spatial resolution of about $30 \mu \mathrm{m}$. Quantitative results for the trace elements reported here were obtained through calibration of relative element sensitivities using NIST-610 standard glass as the external calibration standard. The BCR-2g and zircon 91500 and GJ-1 reference materials were analyzed within the run as an independent control on reproducibility and instrument stability. $\mathrm{Zr}$ content was used for internal calibration for unknown zircon samples $(66$ wt.\% of $\mathrm{ZrO}_{2}$ as the stoichiometric amount of this oxide in zircon). The precision and accuracy of the NIST-610 analyses were determined to be $1-2 \%$ for rare earth elements at the ppm concentration level. Typical detection limits (99\% confidence) are provided in table 4.

\section{RESULTS}

The most common internal feature of sapphire from southern Vietnam was strong color zoning (figure 6A). Other features included parallel twin planes, needle-like inclusions (figure 6B), liquid-filled inclusions (figure 6C), and negative crystals (figure 6D). Based on Raman spectroscopic and EPMA analyses, 290 mineral inclusions were identified; they were mainly $\mathrm{Nb}$-Ta oxides, followed by silicate and other oxide minerals. The types of mineral inclusions in each sample group are summarized in table 1. Mineral inclusions previously reported in sapphires from Thailand, Laos, and Cambodia are also compared in the same table. Columbite and zircon were the most
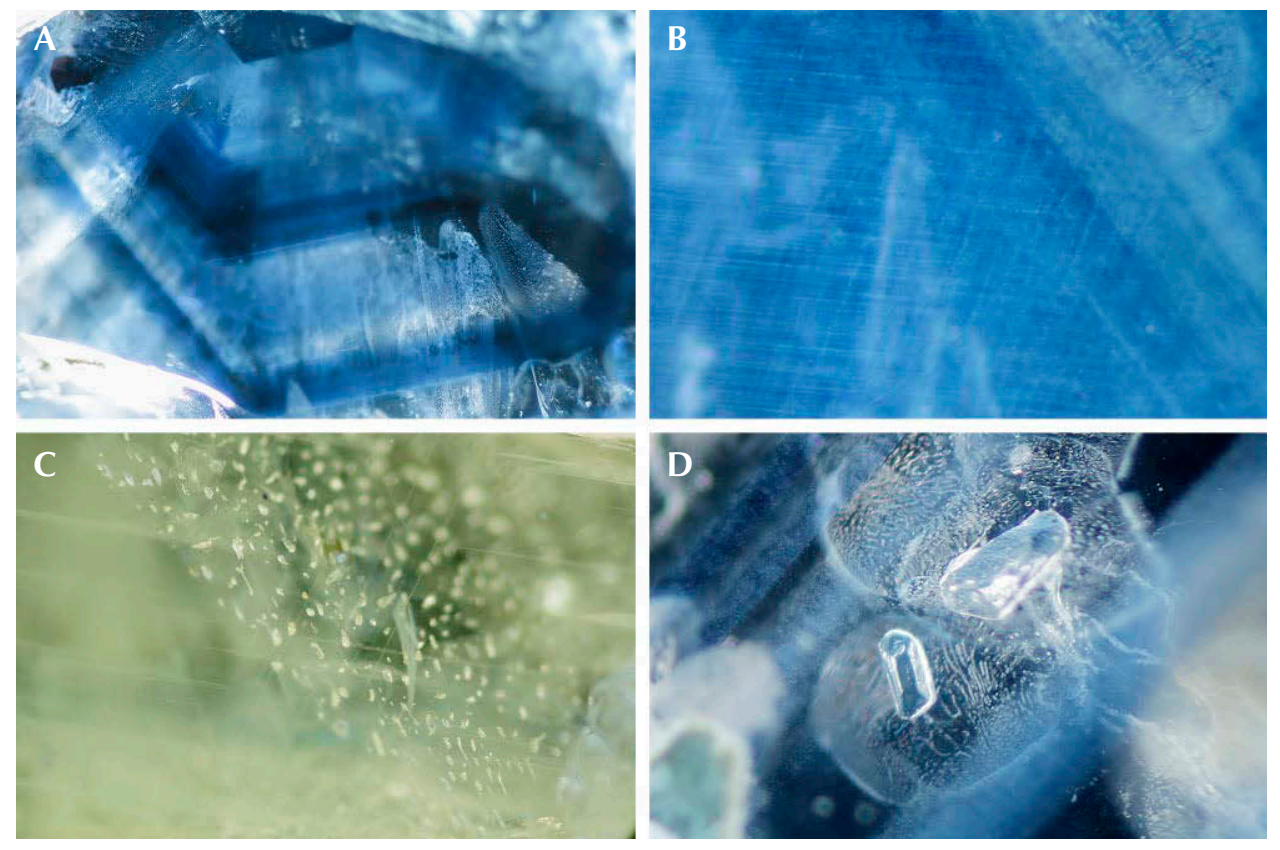

Figure 6. Typical internal features observed in sapphire from southern Vietnam: strong color zones (A), needle-like inclusions (B), liquidfilled inclusions $(C)$, and

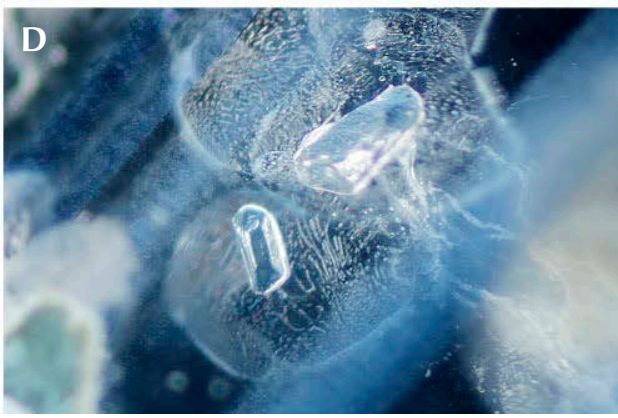
negative crystals situated in healed fractures (D). Darkfield illumination. Photomicrographs by Doan Thi Anh Vu; magnified 50x. 
TABLE 1. Summary of mineral inclusions found in sapphire from southern Vietnam, compared to those reported from basaltic gem fields elsewhere in Southeast Asia.

\begin{tabular}{|c|c|c|c|}
\hline Area & Nb-Ta oxides & Silicate & Other oxides \\
\hline \multicolumn{4}{|c|}{ Southern Vietnam } \\
\hline Dak Nong & $\begin{array}{l}\text { Columbite*a } \\
\text { Pyrochlore* }\end{array}$ & $\begin{array}{l}\text { Zircon*a,b } \\
\text { Alkali feldspar*a }\end{array}$ & $\begin{array}{l}\text { Spinel* } \\
\text { Ilmenite* } \\
\text { Unidentified iron* }\end{array}$ \\
\hline Di Linh & $\begin{array}{l}\text { Columbite } \\
\text { Pyrochlore }^{* c}\end{array}$ & $\begin{array}{l}\text { Zircon*c } \\
\text { Alkali feldspar*c }\end{array}$ & $\begin{array}{l}\text { Spinel*c } \\
\text { Ilmenitec } \\
\text { Unidentified iron* }\end{array}$ \\
\hline Krong Nang & $\begin{array}{l}\text { Columbite* } \\
\text { Pyrochlore* }\end{array}$ & $\begin{array}{l}\text { Zircon* } \\
\text { Alkali feldspar* }\end{array}$ & $\begin{array}{l}\text { Spinel* } \\
\text { Unidentified iron* }\end{array}$ \\
\hline Binh Thuan & $\begin{array}{l}\text { Columbite } \\
\text { Pyrochlore }^{* c}\end{array}$ & $\begin{array}{l}\text { Zircon*b,c } \\
\text { Alkali feldspar*c }\end{array}$ & $\begin{array}{l}\text { Spinel*c } \\
\text { Ilmenitec }^{c} \\
\text { Unidentified iron* }\end{array}$ \\
\hline \multicolumn{4}{|c|}{ Southeast Asia } \\
\hline $\begin{array}{l}\text { Chanthaburi } \\
\text { (Thailand) }\end{array}$ & Columbite $^{d}$ & $\begin{array}{l}\text { Zircond } \\
\text { Alkali feldspar }\end{array}$ & Spineld,e \\
\hline $\begin{array}{l}\text { Kanchanaburi } \\
\text { (Thailand) }\end{array}$ & - & $\begin{array}{l}\text { Zircon }^{f} \\
\text { Alkali feldspar }\end{array}$ & $\begin{array}{l}\text { Spinel } \\
\text { Ilmenite }^{f}\end{array}$ \\
\hline $\begin{array}{l}\text { Ban Huai Sai } \\
\text { (Laos) }\end{array}$ & Columbiteg & $\begin{array}{l}\text { Zircone,h } \\
\text { Alkali feldsparh }\end{array}$ & Spinele \\
\hline $\begin{array}{l}\text { Pailin } \\
\text { (Cambodia) }\end{array}$ & Pyrochlore & - & Spinels \\
\hline \multicolumn{4}{|c|}{$\begin{array}{l}\text { *This study, using Raman and EPMA } \\
\text { a'zokh et al. (2010), using EPMA } \\
\text { 'Long et al. (2004), using SEM-EDS } \\
\text { 'Smith et al. (1995), using SEM-EDS } \\
\text { 'Promwongnan and Sutthirat (2019), using Raman and EPMA } \\
\text { 'Saminpanya and Sutherland (2011), using EPMA } \\
\text { 'Khamloet et al. (2014), using EPMA } \\
\text { 'Sutherland et al. (1998b), using EPMA } \\
\text { "Sutherland et al. (2002), using EPMA } \\
\text { iPalke et al. (2019), using Raman }\end{array}$} \\
\hline
\end{tabular}

common inclusions in these sapphire samples, while alkali feldspar and spinel were often identified. Ilmenite, pyrochlore, and unidentified iron minerals were rarely detected. Details of these inclusions are provided below.

Columbite was the predominant mineral inclusion observed in sapphires from all gem fields in southern Vietnam, accounting for nearly $47 \%$ of the
290 mineral inclusions contained in the 274 samples They generally formed as opaque black euhedral crystals with a rhombic prism with pyramidal shape and a truncated rhombic pyramid shape, ranging in sizes from $<10 \mu \mathrm{m}$ up to several millimeters (figure 7, A and B). They were often associated with zircon, with or without feldspar (figures 7C and 9B). Representative EPMA analyses of columbite inclusions

Figure 7. A: A larger prismatic columbite inclusion. B: Two truncated-rhombic columbite inclusions surrounded by healed fractures. $C$ : A cluster of several tiny columbites with colorless feldspar and zircon inclusions. Darkfield illumination. Photomicrographs by Doan Thi Anh Vu; magnified 50x.
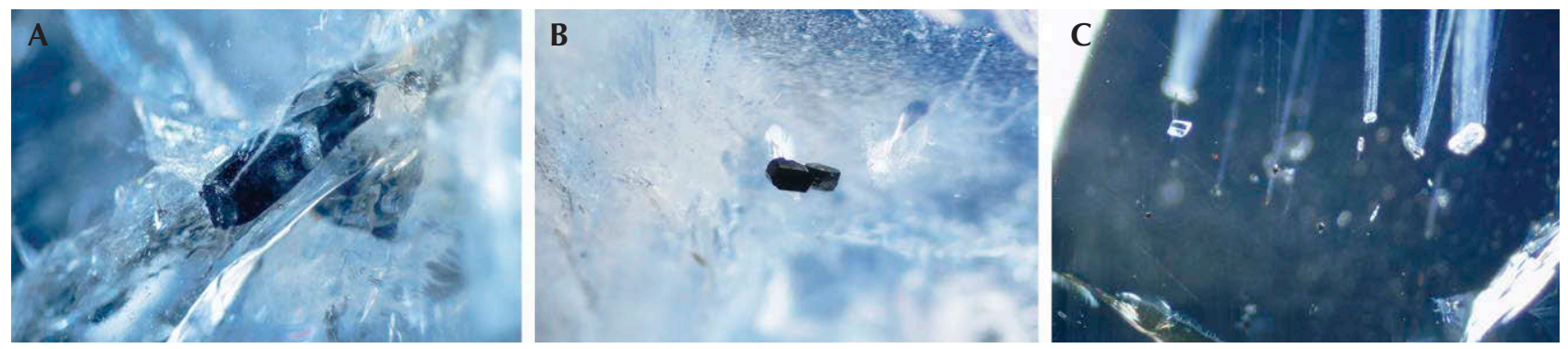


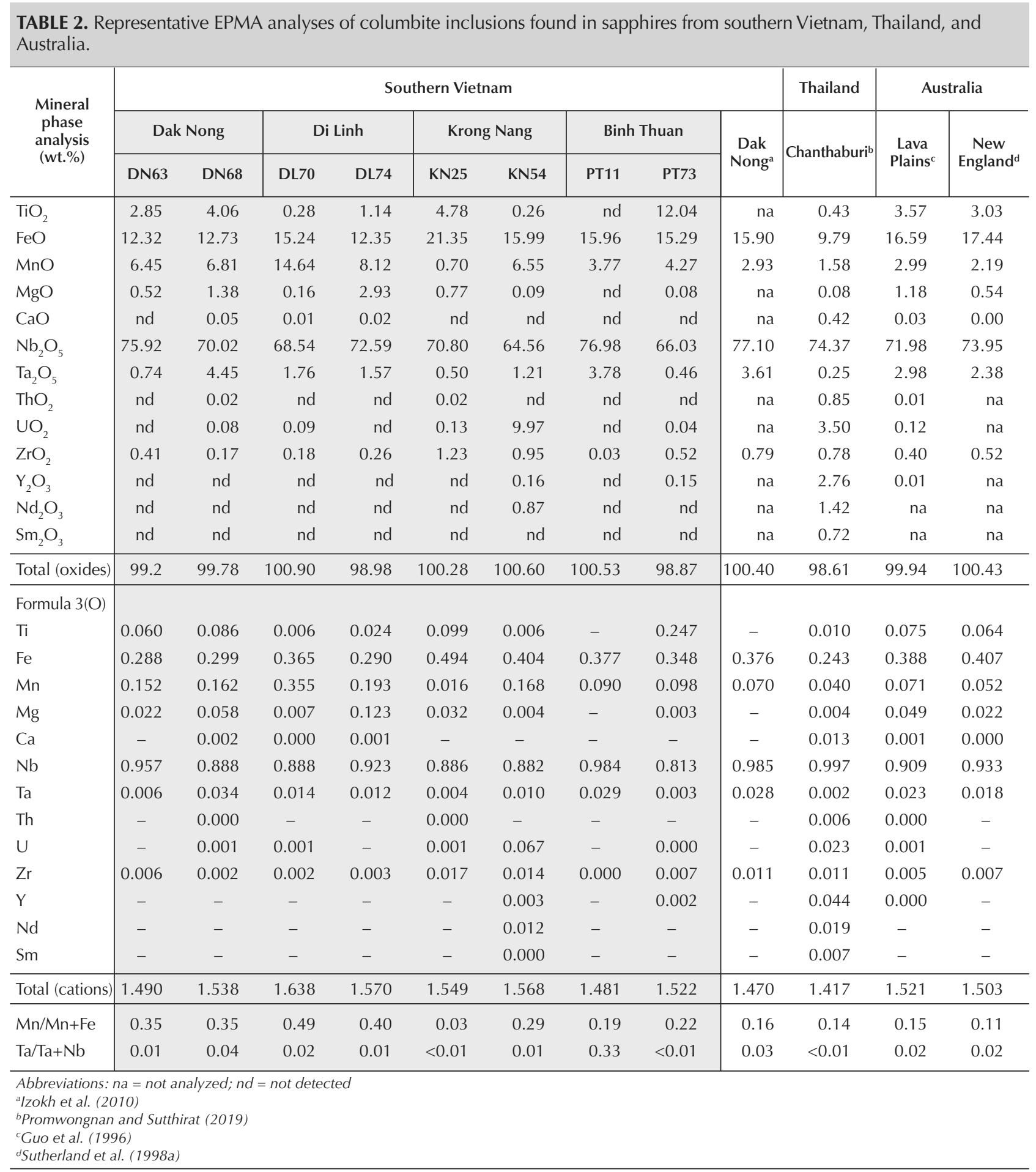

ranged between 65-77 wt. $\% \mathrm{Nb}_{2} \mathrm{O}_{5} \leq 4.5 \mathrm{wt} . \% \mathrm{Ta}_{2} \mathrm{O}_{5}$, $\leq 12$ wt. $\% \mathrm{TiO}_{2}, \leq 3$ wt. $\% \mathrm{MgO}, 12-21$ wt. $\% \mathrm{FeO}$, and $<15 \mathrm{wt} . \% \mathrm{MnO}$ (table 2); The chemical formula of these columbite inclusions can be expressed as $\left(\mathrm{Fe}_{0.373} \mathrm{Mn}_{0.133} \mathrm{Mg}_{0.133} \mathrm{Ca}_{0.228} \mathrm{Ti}_{0.091}\right)\left(\mathrm{Nb}_{0.938} \mathrm{Ta}_{0.010} \mathrm{U}_{0.001} \mathrm{Zr}_{0.007}\right)_{2} \mathrm{O}_{6^{\prime}}$ which fits very well within the compositional range of ferrocolumbite (figure 8). Their compositions were also equivalent to those previously reported for sapphires from Dak Nong by Izokh et al. (2010) as well as from Lava Plains and New England in Australia (Guo et al., 1996; Sutherland et al., 1998b). Lower FeO contents of columbite inclusions in Bo Welu sapphire 


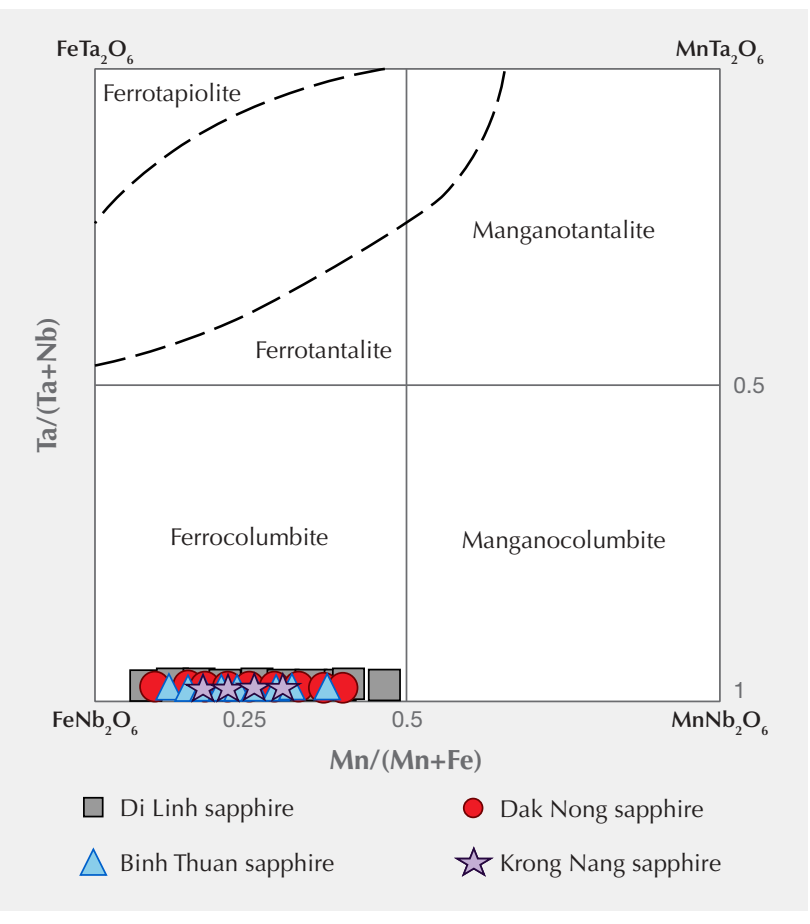

Figure 8. Quadrilateral compositional plots of ferrocolumbite inclusions found in sapphires from southern Vietnam. Various compositional fields and columbite-tapiolite miscibility gap were proposed by Cerný and Ercit (1985).

from Chanthaburi, Thailand, were reported by Promwongnan and Sutthirat (2019). Ferrocolumbite inclusions in sapphire from southern Vietnam contained low $\mathrm{Ta}$ content, yielding a $\mathrm{Ta} /(\mathrm{Ta}+\mathrm{Nb})$ ratio $<0.05$ within a wide range of $\mathrm{Mn}$ contents $(<15 \mathrm{wt} . \% \mathrm{MnO}$ and $\mathrm{Mn} /(\mathrm{Mn}+\mathrm{Fe})$ ratio $<0.5$ ) (figure 8), which may have derived from peralkaline complexes (peralkaline granite/syenite) (Mackay and Simandl, 2015).

Zircon was the second most abundant inclusion, representing about $22 \%$ of the mineral inclusions contained in the sapphire samples. These inclusions commonly formed as euhedral tetragonal prismatic or dipyramidal crystals (figure 9, A and B). Most of the zircons were colorless, but some had orange to orange-red colors. Their chemical compositions varied within narrow ranges of approximately 31-34 wt. $\% \mathrm{SiO}_{2}$ and 61-65 wt. $\% \mathrm{ZrO}_{2}$ (table 3). The hafnium contents showed a slightly wider range of about 1-4 wt.\%, leading to $\mathrm{Hf} / \mathrm{Zr}$ ratios of 0.01 to 0.04 , which fall in the range of magmatic zircon, particularly syenite and granite zircons (Deer et al., 2013). Significant trace elements included $<1.5 \mathrm{wt} . \%$ $\mathrm{ThO}_{2},<1.8 \mathrm{wt}$. $\% \mathrm{UO}_{2}$, and $<0.6 \mathrm{wt} . \% \mathrm{Y}_{2} \mathrm{O}_{3}$; these elements appeared to be higher in zircon inclusions of Di Linh sapphire. On the other hand, these zircon inclusions yielded $\mathrm{Th} / \mathrm{U}$ ratios $>0.2$, indicating a magmatic source (Williams and Claesson, 1987; Rubatto and Gebauer, 2000; Huong et al., 2016). These were similar in composition to zircon analyses previously reported from Dak Nong (Izokh et al., 2010) and other basalt-associated sources such as Ban Huai Sai in Laos, Kanchanaburi in Thailand, and Kings Plains and New England in Australia (Guo et al., 1996; Sutherland et al., 1998a; 2002; Khamloet et al., 2014), although the $\mathrm{Y}_{2} \mathrm{O}_{3}$ content of zircon inclusions from Bo Welu in Thailand (Promwongnan and Sutthirat, 2019) has recently been reported with a higher content. Total REE contents of representative zircon inclusions ranged from 1156 to $2710 \mathrm{ppm}$ (table 4), more similar to those from syenite pegmatite (2043 $\mathrm{ppm})$ and granitoid $(1813 \mathrm{ppm})$ than those from carbonatite (600-700 ppm) (Belousova et al., 2002).

Alkali feldspar inclusions were sometimes observed in the sapphire samples, making up about $13 \%$ of the mineral inclusions. They generally presented subhedral or euhedral grains with small sizes ranging from about $40 \mu \mathrm{m}$, with some rare examples reaching $1 \mathrm{~mm}$. These included crystals were commonly transparent and colorless (figures 7C and 9C).

Figure 9. A: Prismatic orange zircon inclusion. B: Colorless dipyramidal zircon inclusion surrounded by radial cracks, associated with black columbite inclusions. C: Tiny euhedral feldspar inclusions. Darkfield illumination. Photomicrographs by Doan Thi Anh Vu; magnified 50x.
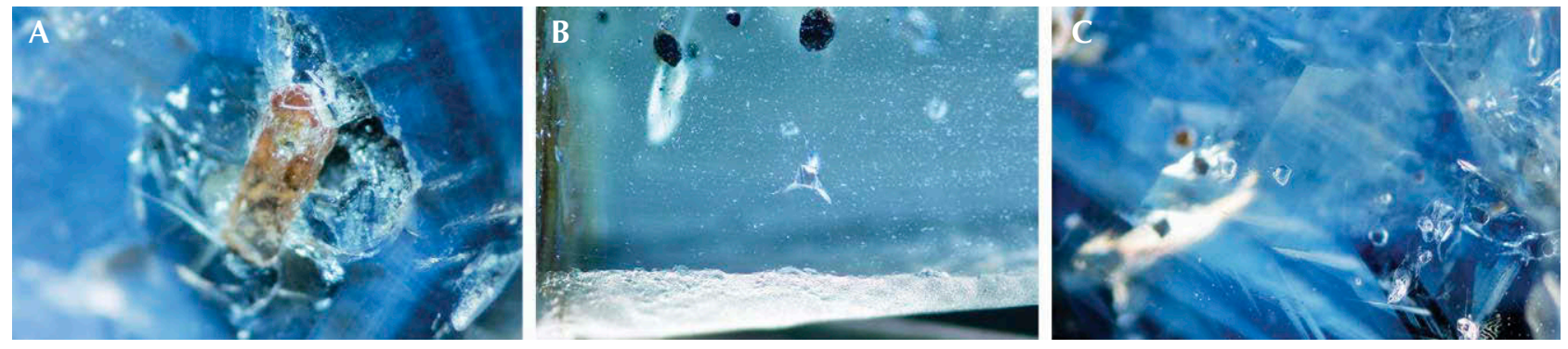


\begin{tabular}{|c|c|c|c|c|c|c|c|c|c|c|c|c|c|c|}
\hline \multirow{3}{*}{$\begin{array}{l}\text { Mineral } \\
\text { phase } \\
\text { analysis } \\
\text { (wt.\%) }\end{array}$} & \multicolumn{9}{|c|}{ Southern Vietnam } & \multirow{3}{*}{\begin{tabular}{|c|} 
Laos \\
$\begin{array}{c}\text { Ban Huai } \\
\text { Sai }\end{array}$
\end{tabular}} & \multicolumn{2}{|c|}{ Thailand } & \multicolumn{2}{|c|}{ Australia } \\
\hline & \multicolumn{2}{|c|}{ Dak Nong } & \multicolumn{2}{|c|}{ Di Linh } & \multicolumn{2}{|c|}{ Krong Nang } & \multicolumn{2}{|c|}{ Binh Thuan } & \multirow{2}{*}{$\begin{array}{c}\text { Dak } \\
\text { Nong }^{\mathrm{a}}\end{array}$} & & \multirow{2}{*}{$\begin{array}{c}\text { Chantha- } \\
\text { buric }^{-}\end{array}$} & \multirow{2}{*}{$\begin{array}{l}\text { Kancha- } \\
\text { naburid }^{d}\end{array}$} & \multirow{2}{*}{$\begin{array}{l}\text { Kings } \\
\text { Plains }^{\mathrm{e}}\end{array}$} & \multirow{2}{*}{$\begin{array}{c}\text { New } \\
\text { England }\end{array}$} \\
\hline & DN23 & DN31 & DL18 & DL44 & KN15 & KN13 & PT36 & PT65 & & & & & & \\
\hline $\mathrm{SiO}_{2}$ & 32.16 & 32.43 & 34.34 & 32.59 & 33.84 & 33.43 & 31.19 & 33.86 & 32.34 & 31.23 & 33.63 & 34.14 & 32.12 & 31.48 \\
\hline $\mathrm{TiO}_{2}$ & 0.03 & 0.02 & nd & 0.01 & 0.01 & nd & 0.06 & nd & na & na & na & na & $<0.01$ & 0.00 \\
\hline $\mathrm{Al}_{2} \mathrm{O}_{3}$ & 0.27 & 0.30 & nd & nd & nd & nd & 0.28 & 0.01 & na & 0.01 & 0.01 & 0.17 & 0.02 & 0.44 \\
\hline $\mathrm{FeO}$ & nd & 0.02 & 0.04 & nd & nd & 0.10 & 0.31 & nd & na & na & 0.07 & 0.04 & 0.20 & 0.13 \\
\hline $\mathrm{MgO}$ & 0.02 & 0.01 & nd & nd & nd & nd & 0.02 & 0.01 & na & 0.01 & na & na & $<0.01$ & 0.00 \\
\hline $\mathrm{CaO}$ & 0.06 & 0.05 & nd & nd & nd & 0.01 & 0.01 & nd & na & na & na & 0.00 & 0.02 & 0.00 \\
\hline $\mathrm{Na}_{2} \mathrm{O}$ & nd & nd & nd & nd & nd & nd & 0.04 & nd & na & na & na & na & na & 0.00 \\
\hline $\mathrm{K}_{2} \mathrm{O}$ & 0.02 & nd & nd & nd & nd & 0.05 & 0.67 & nd & na & na & na & na & na & 0.00 \\
\hline $\mathrm{ThO}_{2}$ & 0.03 & 0.31 & 0.11 & 1.40 & 0.32 & 0.03 & 0.13 & 0.09 & 0.76 & 0.18 & 1.21 & na & 0.49 & 0.11 \\
\hline $\mathrm{UO}_{2}$ & 0.12 & 1.40 & 0.12 & 1.73 & 0.23 & 0.06 & 0.17 & 0.34 & 0.93 & 0.41 & 1.13 & na & 0.84 & 0.11 \\
\hline $\mathrm{ZrO}_{2}$ & 64.47 & 61.44 & 62.20 & 61.00 & 63.98 & 63.29 & 63.78 & 62.02 & 63.96 & 64.02 & 59.39 & 62.40 & 63.78 & 64.84 \\
\hline $\mathrm{HfO}_{2}$ & 1.62 & 3.70 & 2.51 & 3.08 & 2.00 & 2.82 & 2.62 & 3.85 & 2.44 & 3.58 & 2.62 & 3.50 & 2.88 & 2.58 \\
\hline $\mathrm{P}_{2} \mathrm{O}_{5}$ & 0.13 & 0.16 & nd & 0.22 & 0.08 & 0.09 & 0.32 & 0.23 & na & 0.25 & 0.57 & na & 0.34 & na \\
\hline $\mathrm{Y}_{2} \mathrm{O}_{3}$ & 0.02 & 0.08 & 0.27 & 0.56 & 0.05 & 0.07 & 0.28 & 0.03 & na & 0.80 & 0.85 & na & 0.34 & 0.46 \\
\hline Total (oxides) & 98.93 & 99.91 & 99.60 & 100.58 & 100.52 & 99.96 & 99.87 & 100.42 & 100.43 & 100.55 & 99.57 & 100.25 & 101.04 & 100.15 \\
\hline \multicolumn{15}{|l|}{ Formula $4(\mathrm{O})$} \\
\hline Si & 0.996 & 1.008 & 1.048 & 1.013 & 1.028 & 1.024 & 0.971 & 1.033 & 1.001 & 0.973 & 1.035 & 1.036 & 0.989 & 0.976 \\
\hline $\mathrm{Ti}$ & 0.001 & 0.000 & - & 0.000 & 0.000 & - & 0.001 & - & - & - & - & - & - & 0.000 \\
\hline $\mathrm{Al}$ & 0.010 & 0.011 & - & - & - & - & 0.010 & 0.000 & - & 0.000 & 0.000 & 0.006 & 0.001 & 0.016 \\
\hline $\mathrm{Fe}$ & - & 0.000 & 0.001 & - & - & 0.003 & 0.008 & - & - & - & 0.002 & 0.001 & 0.046 & 0.003 \\
\hline$M g$ & 0.001 & 0.001 & - & - & - & - & 0.001 & 0.000 & - & 0.000 & - & - & - & 0.000 \\
\hline $\mathrm{Ca}$ & 0.002 & 0.002 & - & - & - & 0.000 & 0.000 & - & - & - & - & 0.000 & 0.001 & 0.000 \\
\hline $\mathrm{Na}$ & - & - & - & - & - & - & 0.003 & - & - & - & - & - & - & 0.000 \\
\hline K & 0.001 & - & - & - & - & 0.002 & 0.027 & - & - & - & - & - & - & 0.000 \\
\hline Th & 0.000 & 0.002 & 0.001 & 0.010 & 0.002 & 0.000 & 0.001 & 0.001 & 0.005 & 0.001 & 0.008 & - & 0.003 & 0.001 \\
\hline$U$ & 0.001 & 0.010 & 0.001 & 0.012 & 0.002 & 0.000 & 0.001 & 0.002 & 0.006 & 0.003 & 0.008 & - & 0.006 & 0.001 \\
\hline $\mathrm{Zr}$ & 0.974 & 0.931 & 0.925 & 0.924 & 0.948 & 0.945 & 0.968 & 0.923 & 0.965 & 0.972 & 0.891 & 0.923 & 0.957 & 0.980 \\
\hline $\mathrm{Hf}$ & 0.014 & 0.033 & 0.022 & 0.027 & 0.017 & 0.025 & 0.023 & 0.033 & 0.022 & 0.032 & 0.023 & 0.036 & 0.025 & 0.023 \\
\hline P & 0.003 & 0.004 & - & 0.006 & 0.002 & 0.002 & 0.008 & 0.006 & - & 0.007 & 0.013 & - & 0.009 & - \\
\hline Y & 0.000 & 0.001 & 0.004 & 0.009 & 0.001 & 0.001 & 0.005 & 0.000 & - & 0.013 & 0.014 & - & 0.006 & 0.008 \\
\hline Total (cations) & 2.004 & 2.003 & 2.002 & 2.001 & 2.000 & 2.003 & 2.028 & 1.999 & 2.000 & 2.002 & 1.995 & 2.002 & 2.001 & 2.008 \\
\hline Th/U & 0.29 & 0.23 & 0.93 & 0.83 & 1.42 & 0.51 & 0.79 & 0.26 & 0.84 & 0.45 & 1.10 & - & 0.60 & 1.02 \\
\hline $\mathrm{Hf} / \mathrm{Zr}$ & 0.02 & 0.04 & 0.02 & 0.03 & 0.02 & 0.03 & 0.02 & 0.04 & 0.02 & 0.03 & 0.03 & 0.03 & 0.03 & 0.02 \\
\hline \multicolumn{15}{|c|}{$\begin{array}{l}\text { Abbreviations: } n a=\text { not analyzed; } n d=\text { not detected } \\
\text { alzokh et al. (2010) }\end{array}$} \\
\hline
\end{tabular}

Their chemical compositions were rather uniformly toward Na-rich feldspar between albite-anorthoclaseoligoclase $\left(\mathrm{Ab}_{77-98} \mathrm{An}_{<15} \mathrm{Or}_{1-11}\right.$, see table 5 and figure
10). However, feldspar in Krong Nang sapphires mostly plotted close to the junction of albite-anorthoclase-oligoclase compositions $\left(\mathrm{Ab}_{82-86} \mathrm{An}_{6-11} \mathrm{Or}_{6-11}\right)_{\text {, }}$ 
TABLE 4. REE analyses (ppm) of representative zircons found in southern Vietnam sapphire, determined by LA-ICP-MS.

\begin{tabular}{|c|c|c|c|c|c|}
\hline \multirow{2}{*}{ Element } & Dak Nong & Di Linh & $\begin{array}{l}\text { Krong } \\
\text { Nang }\end{array}$ & $\begin{array}{l}\text { Binh } \\
\text { Thuan }\end{array}$ & \multirow{2}{*}{$\begin{array}{l}\text { Typical detection } \\
\quad \text { limits } \\
(99 \% \text { confidence })\end{array}$} \\
\hline & DN05 & DL46 & KN08 & РT05 & \\
\hline $\mathrm{Y}$ & 1468.21 & 4220.35 & 1194.74 & 2868.13 & 0.004 \\
\hline $\mathrm{La}$ & 0.02 & nd & 0.01 & nd & 0.004 \\
\hline $\mathrm{Ce}$ & 2.79 & 12.00 & 1.65 & 13.33 & 0.003 \\
\hline $\operatorname{Pr}$ & 0.01 & 0.06 & 0.02 & 0.0523 & 0.003 \\
\hline $\mathrm{Nd}$ & 0.27 & 1.11 & 0.26 & 1.451 & 0.014 \\
\hline $\mathrm{Sm}$ & 1.23 & 5.62 & 0.68 & 5.62 & 0.019 \\
\hline $\mathrm{Eu}$ & 0.96 & 5.12 & 0.59 & 2.177 & 0.006 \\
\hline Gd & 12.91 & 54.66 & 5.74 & 44.06 & 0.019 \\
\hline $\mathrm{Tb}$ & 7.23 & 27.58 & 3.91 & 21.66 & 0.003 \\
\hline Dy & 122.00 & 416.17 & 76.25 & 310.13 & 0.013 \\
\hline $\mathrm{Ho}$ & 50.02 & 152.71 & 38.25 & 104.29 & 0.003 \\
\hline $\mathrm{Er}$ & 258.30 & 672.98 & 258.42 & 442.14 & 0.009 \\
\hline $\mathrm{Tm}$ & 60.40 & 134.11 & 83.73 & 89.21 & 0.003 \\
\hline $\mathrm{Yb}$ & 566.33 & 115.50 & 1081.3 & 721.34 & 0.018 \\
\hline Lu & 80.77 & 140.18 & 179.34 & 81.68 & 0.003 \\
\hline Total REE & 1155.94 & 2710.22 & 1762.23 & 1815.47 & \\
\hline
\end{tabular}

whereas Di Linh feldspar was divided into two groups: the first group close to the albite-anorthoclase boundary $\left(\mathrm{Ab}_{82-85} \mathrm{An}_{6-9} \mathrm{Or}_{8-11}\right)$ and the second
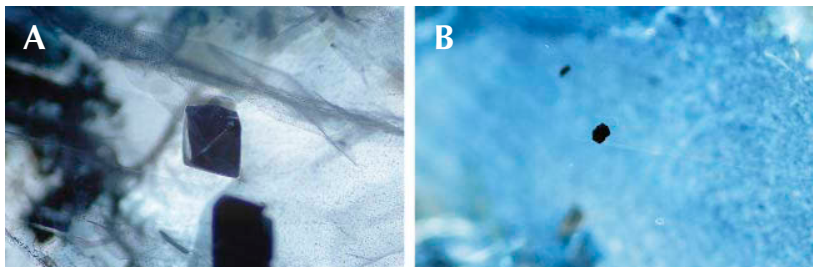

Figure 11. Inclusions of black octahedral hercynite spinel (A) and euhedral rhombohedral ilmenite (B). Darkfield illumination. Photomicrographs by Doan Thi Anh Vu; magnified 50x.

one containing lower $\mathrm{K}$ content near the albite-oligoclase boundary $\left(\mathrm{Ab}_{85-87} \mathrm{An}_{7-11} \mathrm{Or}_{4-5}\right)$. Binh Thuan feldspar ranged between albite and oligoclase compositions, while most Dak Nong feldspar fell within albite composition $\left(\mathrm{Ab}_{83-98} \mathrm{An}_{1-10} \mathrm{Or}_{1-8}\right)$. These compositional ranges were wider than those previously reported, as only oligoclase was identified in sapphires from Dak Nong (Izokh et al., 2010) and from Ban Huai Sai in Laos (Sutherland et al., 2002), but An and Or components were narrower in range than those in Thai sapphires from Kanchanaburi $\left(\mathrm{An}_{9}\right.$ ${ }_{23} \mathrm{Or}_{6-14}$ ) (Khamloet et al., 2014) and Chanthaburi $\left(\mathrm{An}_{<14} \mathrm{Or}_{7-17}\right)($ Promwongnan and Sutthirat, 2019) and Australian sapphires from Kings Plains $\left(\mathrm{An}_{<14} \mathrm{Or}_{7-17}\right)$

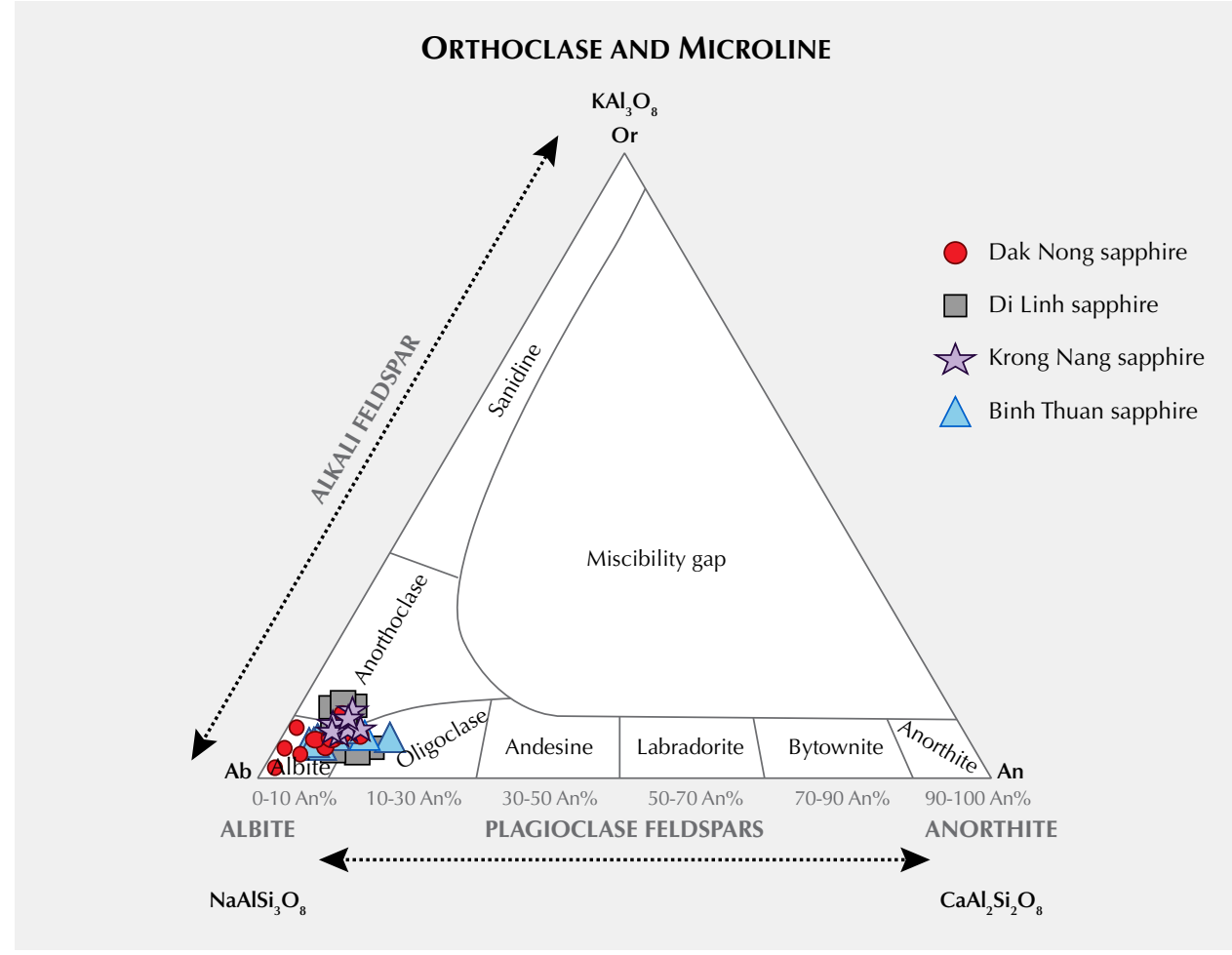

Figure 10. Ternary plot of An-Ab-Or in alkali feldspar inclusions in sapphires from southern Vietnam. Modified from N.N. Greenwood and A. Earnshaw, Chemistry of Elements (1998), p. 357. 
TABLE 5. Representative EPMA analyses of feldspar inclusions in sapphires from southern Vietnam, Laos, Thailand, and Australia.

\begin{tabular}{|c|c|c|c|c|c|c|c|c|c|c|c|c|c|c|}
\hline \multirow{3}{*}{$\begin{array}{l}\text { Mineral } \\
\text { phase } \\
\text { analysis } \\
\text { (wt.\%) }\end{array}$} & \multicolumn{9}{|c|}{ Southern Vietnam } & \multirow{3}{*}{\begin{tabular}{|c|} 
Laos \\
$\begin{array}{c}\text { Ban Huai } \\
\text { Sai }^{b}\end{array}$
\end{tabular}} & \multicolumn{2}{|c|}{ Thailand } & \multicolumn{2}{|c|}{ Australia } \\
\hline & \multicolumn{2}{|c|}{ Dak Nong } & \multicolumn{2}{|c|}{ Di Linh } & \multicolumn{2}{|c|}{ Krong Nang } & \multicolumn{2}{|c|}{ Binh Thuan } & \multirow{2}{*}{$\begin{array}{l}\text { Dak } \\
\text { Nong }^{\mathrm{a}}\end{array}$} & & \multirow{2}{*}{$\begin{array}{l}\text { Kancha- } \\
\text { naburic }\end{array}$} & \multirow{2}{*}{$\begin{array}{c}\text { Chantha- } \\
\text { burid }^{\mathrm{d}}\end{array}$} & \multirow{2}{*}{$\begin{array}{l}\text { Kings } \\
\text { Plains }\end{array}$} & \multirow{2}{*}{$\begin{array}{c}\text { New } \\
\text { England }\end{array}$} \\
\hline & DN41 & DN34 & DL27 & DL34 & KN53 & KN37 & РT01 & PT23 & & & & & & \\
\hline $\mathrm{SiO}_{2}$ & 67.93 & 64.13 & 65.66 & 62.30 & 65.83 & 64.38 & 68.89 & 64.31 & 64.20 & 64.83 & 65.60 & 66.97 & 64.81 & 53.47 \\
\hline $\mathrm{TiO}_{2}$ & 0.02 & nd & 0.01 & 0.02 & 0.01 & 0.01 & nd & nd & 0.00 & na & 0.01 & 0.02 & na & 0.00 \\
\hline $\mathrm{Al}_{2} \mathrm{O}_{3}$ & 19.06 & 21.92 & 23.72 & 23.73 & 22.86 & 21.79 & 19.70 & 22.39 & 22.10 & 21.45 & 21.50 & 21.40 & 18.56 & 29.28 \\
\hline $\mathrm{FeO}$ & 0.03 & 0.06 & 0.03 & 0.19 & 0.09 & 0.07 & nd & 0.06 & 0.06 & 0.03 & 0.04 & 0.65 & na & 0.09 \\
\hline $\mathrm{MnO}$ & nd & nd & nd & nd & nd & nd & nd & nd & 0.02 & na & 0.00 & 0.04 & na & 0.00 \\
\hline $\mathrm{MgO}$ & nd & nd & nd & nd & nd & nd & nd & nd & 0.00 & na & 0.05 & nd & na & 0.00 \\
\hline $\mathrm{BaO}$ & 0.07 & 0.01 & 0.03 & 0.06 & 0.07 & nd & 0.02 & 0.03 & na & na & na & na & na & na \\
\hline $\mathrm{CaO}$ & 0.17 & 2.24 & 1.09 & 1.64 & 1.54 & 2.24 & nd & 2.98 & 3.13 & 1.96 & 2.59 & 0.73 & $<0.01$ & 11.19 \\
\hline $\mathrm{Na}_{2} \mathrm{O}$ & 11.33 & 9.65 & 7.63 & 10.76 & 8.73 & 9.22 & 11.37 & 9.02 & 9.71 & 9.37 & 4.31 & 2.69 & 0.73 & 5.50 \\
\hline $\mathrm{K}_{2} \mathrm{O}$ & 0.22 & 1.15 & 1.60 & 1.02 & 1.70 & 1.44 & 0.32 & 1.43 & 0.82 & 1.53 & 6.52 & 8.47 & 15.50 & 0.48 \\
\hline Total (oxides) & 98.83 & 99.16 & 99.76 & 99.72 & 100.84 & 99.15 & 100.31 & 100.22 & 100.10 & 99.17 & 100.62 & 100.97 & 99.60 & 100.01 \\
\hline \multicolumn{15}{|l|}{ Formula $8(\mathrm{O})$} \\
\hline $\mathrm{Si}$ & 3.003 & 2.857 & 2.872 & 2.775 & 2.872 & 2.868 & 2.998 & 2.841 & 2.840 & 2.885 & 2.908 & 2.957 & 2.997 & 2.425 \\
\hline $\mathrm{Ti}$ & 0.001 & - & 0.000 & 0.001 & 0.000 & 0.000 & - & - & - & - & 0.000 & 0.001 & - & - \\
\hline $\mathrm{Al}$ & 0.993 & 1.151 & 1.223 & 1.245 & 1.175 & 1.144 & 1.010 & 1.166 & 1.152 & 1.125 & 1.124 & 1.114 & 1.011 & 1.565 \\
\hline $\mathrm{Fe}$ & 0.001 & 0.002 & 0.001 & 0.007 & 0.003 & 0.003 & - & 0.002 & 0.002 & 0.001 & 0.002 & 0.024 & - & 0.003 \\
\hline Mn & - & - & - & - & - & - & - & - & 0.001 & - & - & 0.001 & - & - \\
\hline $\mathrm{Mg}$ & - & - & - & - & - & - & - & - & - & - & 0.003 & 0.000 & - & - \\
\hline $\mathrm{Ba}$ & 0.001 & 0.000 & 0.000 & 0.001 & 0.001 & - & 0.000 & 0.001 & - & - & - & 0.000 & - & - \\
\hline $\mathrm{Ca}$ & 0.008 & 0.107 & 0.051 & 0.078 & 0.072 & 0.107 & - & 0.141 & 0.148 & 0.093 & 0.123 & 0.035 & - & 0.544 \\
\hline $\mathrm{Na}$ & 0.971 & 0.833 & 0.647 & 0.929 & 0.738 & 0.796 & 0.959 & 0.772 & 0.833 & 0.808 & 0.371 & 0.230 & 0.065 & 0.484 \\
\hline K & 0.012 & 0.065 & 0.089 & 0.058 & 0.095 & 0.082 & 0.018 & 0.081 & 0.046 & 0.087 & 0.369 & 0.477 & 0.914 & 0.028 \\
\hline Total (cations) & 4.991 & 5.016 & 4.884 & 5.095 & 4.957 & 4.999 & 4.986 & 5.003 & 5.023 & 5.000 & 4.899 & 4.893 & 4.988 & 5.048 \\
\hline \multicolumn{15}{|l|}{ Atomic (\%) } \\
\hline Albite & 97.9 & 82.9 & 82.1 & 87.2 & 81.6 & 80.8 & 98.2 & 77.7 & 81.1 & 81.8 & 43.0 & 31.0 & 6.7 & 45.8 \\
\hline Anorthite & 0.8 & 10.6 & 6.5 & 7.4 & 8.0 & 10.9 & 0.0 & 14.2 & 14.4 & 9.5 & 14.2 & 4.7 & 0.0 & 51.5 \\
\hline Orthoclase & 1.3 & 6.5 & 11.3 & 5.4 & 10.5 & 8.3 & 1.8 & 8.1 & 4.5 & 8.8 & 42.8 & 64.3 & 93.3 & 2.7 \\
\hline $\begin{array}{l}\text { Abbreviations: } \\
\text { abokh et al. ( } 20 \\
\text { bSutherland et a } \\
\text { "Khamloet et al } \\
\text { dPromwongnan } \\
\text { eGuo et al. (195 } \\
\text { 'Sutherland et a }\end{array}$ & $\begin{array}{l}n a=n o t \\
\text { 10) } \\
\text { l. (2002) } \\
\text { (2014) } \\
\text { and Sutt } \\
\text { 96) } \\
\text { l. (1998a }\end{array}$ & analyzed; & $n d=n o t$ & letected & & & & & & & & & & \\
\hline
\end{tabular}

(Guo et al., 1996) and New England $\left(\mathrm{An}_{<53} \mathrm{Or}_{22-94}\right)$ (Sutherland et al., 1998a).

Spinel inclusions accounted for about $4 \%$ of the mineral inclusions contained in the samples from southern Vietnam. They usually occurred as black cubic crystals (figure 11A). Spinel inclusions with compositions ranging between chromite-hercynite and magnetite-hercynite have been previously reported, on the basis of SEM-EDS analyses, in sapphires from Binh Thuan and Di Linh (Smith et al., 1995). However, EPMA analyses of spinel inclusions in this study revealed significant components of hercynite $\left(\mathrm{FeAl}_{2} \mathrm{O}_{4}, \mathrm{Hc}_{78-100}\right)$ and spinel $\left(\mathrm{MgAl}_{2} \mathrm{O}_{4}, \mathrm{Sp}_{-23}\right)$, whereas other components were negligible (table 6). The spinel inclusions $\mathrm{Mg}^{\prime} \mathrm{Fe}^{2+}$ ratios were mostly less than 1:4, falling into hercynite spinel (Deer et al., 2013). In comparison, these hercynite spinel inclusions contained a moderately lower spinel composition than those in 
TABLE 6. Representative EPMA analyses of spinel inclusions in sapphires from southern Vietnam, Thailand, Cambodia, and Australia.

\begin{tabular}{|c|c|c|c|c|c|c|c|c|c|c|}
\hline \multirow{3}{*}{$\begin{array}{l}\text { Mineral } \\
\text { phase } \\
\text { analysis } \\
\text { (wt.\%) }\end{array}$} & \multicolumn{7}{|c|}{ Southern Vietnam } & \multirow{3}{*}{\begin{tabular}{|c} 
Thailand \\
Kanchanaburia
\end{tabular}} & \multirow{3}{*}{\begin{tabular}{|c|} 
Cambodia \\
Pailin $^{\mathrm{b}}$
\end{tabular}} & \multirow{3}{*}{$\begin{array}{l}\text { Australia } \\
\text { New } \\
\text { Englandc }\end{array}$} \\
\hline & \multicolumn{2}{|c|}{ Dak Nong } & \multirow{2}{*}{$\begin{array}{c}\text { Di Linh } \\
\text { DL62 }\end{array}$} & \multicolumn{2}{|c|}{ Krong Nang } & \multicolumn{2}{|c|}{ Binh Thuan } & & & \\
\hline & DN12 & DN16 & & KN42 & KN47 & PT22 & PT34 & & & \\
\hline $\mathrm{SiO}_{2}$ & nd & nd & 0.01 & nd & nd & nd & 0.25 & 0.03 & 0.03 & 0.00 \\
\hline $\mathrm{TiO}_{2}$ & 0.12 & 0.08 & 0.70 & 0.13 & 0.18 & nd & 1.91 & 0.07 & 0.26 & 0.13 \\
\hline $\mathrm{Al}_{2} \mathrm{O}_{3}$ & 60.99 & 60.93 & 60.76 & 60.31 & 60.11 & 61.18 & 61.58 & 59.52 & 63.00 & 57.04 \\
\hline $\mathrm{Cr}_{2} \mathrm{O}_{3}$ & nd & nd & 0.09 & nd & nd & nd & nd & 0.16 & 0.29 & 0.00 \\
\hline $\mathrm{FeO}_{\text {total }}$ & 33.99 & 34.18 & 33.87 & 34.96 & 33.77 & 33.35 & 35.53 & 35.39 & 21.77 & 34.66 \\
\hline $\mathrm{MnO}$ & nd & 0.89 & 0.34 & 0.05 & 1.18 & 0.53 & 1.44 & 0.22 & 0.15 & 0.48 \\
\hline $\mathrm{MgO}$ & 4.02 & 4.29 & 4.62 & 3.17 & 4.31 & 4.60 & 0.08 & 5.85 & 13.50 & 6.09 \\
\hline $\mathrm{ZnO}$ & 0.12 & 0.38 & 0.18 & nd & 0.51 & 0.93 & 0.07 & 0.14 & na & 0.69 \\
\hline $\mathrm{CaO}$ & 0.06 & nd & nd & nd & nd & nd & nd & 0.00 & $<0.005$ & 0.00 \\
\hline Total (oxides) & 99.29 & 100.74 & 100.58 & 98.61 & 100.06 & 100.58 & 100.95 & 101.36 & 99.00 & 99.15 \\
\hline \multicolumn{11}{|l|}{ Formula 32(O) } \\
\hline $\mathrm{Si}$ & - & - & 0.002 & - & - & - & 0.056 & 0.007 & 0.006 & 0.00 \\
\hline $\mathrm{Ti}$ & 0.020 & 0.014 & 0.117 & 0.023 & 0.030 & - & 0.321 & 0.011 & 0.042 & 0.022 \\
\hline $\mathrm{Al}$ & 16.134 & 15.975 & 15.886 & 16.153 & 15.881 & 16.018 & 16.194 & 15.570 & 15.774 & 15.364 \\
\hline $\mathrm{Cr}$ & - & - & 0.016 & - & - & - & - & 0.028 & 0.049 & - \\
\hline $\mathrm{Fe}^{3+}$ & 0.000 & 0.000 & 0.000 & 0.000 & 0.078 & 0.000 & 0.000 & 0.412 & 0.000 & 0.779 \\
\hline $\mathrm{Fe}^{2+}$ & 6.380 & 6.359 & 6.285 & 6.645 & 6.253 & 6.196 & 6.630 & 6.157 & 3.868 & 5.846 \\
\hline $\mathrm{Mn}$ & - & 0.168 & 0.064 & 0.009 & 0.224 & 0.100 & 0.272 & 0.041 & 0.027 & 0.093 \\
\hline $\mathrm{Mg}$ & 1.344 & 1.421 & 1.529 & 1.072 & 1.440 & 1.524 & 0.026 & 1.934 & 4.276 & 2.075 \\
\hline $\mathrm{Zn}$ & 0.020 & 0.062 & 0.030 & - & 0.122 & 0.152 & 0.012 & 0.023 & - & 0.116 \\
\hline $\mathrm{Ca}$ & 0.015 & - & - & - & - & - & - & - & - & - \\
\hline Total (cations) & 23.913 & 23.999 & 23.929 & 23.901 & 24.029 & 23.991 & 23.526 & 24.183 & 24.041 & 24.296 \\
\hline$\Sigma R^{2+}$ & 7.759 & 8.010 & 7.907 & 7.726 & 8.040 & 7.973 & 6.956 & 8.155 & 8.171 & 8.131 \\
\hline$\Sigma \mathrm{R}^{3+}$ & 16.154 & 15.989 & 16.022 & 16.175 & 15.989 & 16.018 & 16.571 & 16.028 & 15.870 & 16.165 \\
\hline \multicolumn{11}{|l|}{ Atomic (\%) } \\
\hline Spinel & 17.3 & 17.7 & 19.3 & 22.5 & 13.9 & 19.1 & 0.4 & 23.9 & 52.5 & 26.2 \\
\hline Hercynite & 82.2 & 79.4 & 79.5 & 77.5 & 86.1 & 80.3 & 99.6 & 76.1 & 47.5 & 73.8 \\
\hline \multicolumn{11}{|c|}{ 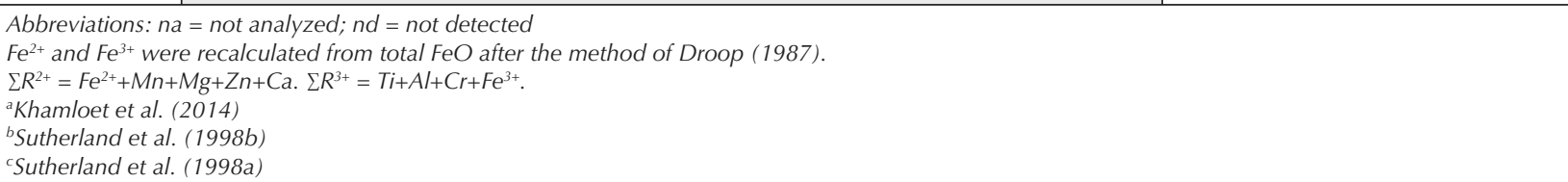 } \\
\hline
\end{tabular}

sapphires from Pailin in Cambodia or from New England in Australia, but nearly the same as those from Kanchanaburi in Thailand (see also table 6).

Ilmenite $\left(\mathrm{FeTiO}_{3}\right)$ was found in only two samples from Di Linh and one sample from Dak Nong. These inclusions were black, opaque, and euhedral (figure 11B), and their identity was confirmed by Raman spectroscopic identification. A previous study, based on SEM-EDS analysis, also recognized ilmenite in sapphires from Di Linh and Binh Thuan (Smith et al.,
1995). The chemical composition of the ilmenite inclusions in this study clearly belonged to the titanohematite series (ilmenite-hematite, $\mathrm{Il}_{54-49} \mathrm{He}_{40-34}$ ) (table 7). The low Mn content (<0.15 wt. \% MnO) of these titanohematites was closer to that from an igneous magma source (Lindsley, 1991) as reported from Sybille Monzosyenite (Fuhrman et al., 1988), and different from ilmenite with higher Mn originating from metamorphic rocks from Western Australia (Cassidy et al., 1988). Moreover, their Mn contents 

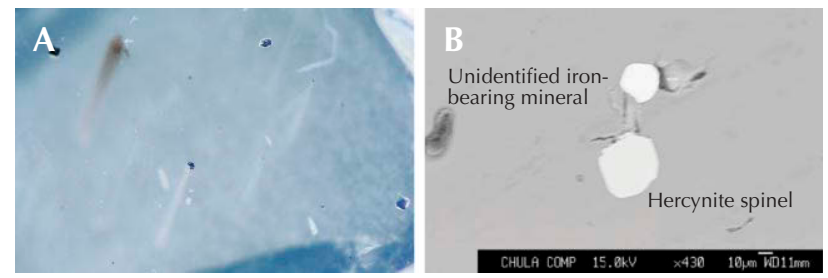

Figure 12. A: Several tiny unidentified iron-bearing minerals with cubic shape, shown in darkfield illumination. Photomicrograph by Doan Thi Anh Vu; magnified 50x. B: Backscattered electron image of unidentified cubic iron oxides and euhedral hercynite-spinel.

were similar to those of ilmenite in New England sapphires originating from silicate melt (Sutherland et al., 1998a) and different from those of ilmenite in Kanchanaburi sapphires from melt involved by subsequent contact metamorphism (Khamloet et al., 2014) (table 7).

Unidentified iron-bearing minerals were also observed in these sapphire samples. They presented as tiny black cubic crystals (less than $10 \mu \mathrm{m}$ and a few around $30 \mu \mathrm{m}$ ) (figure 12A) with morphological forms similar to those identified for spinel inclusions. They were commonly associated with spinel inclusions and sometimes formed as composite inclusions (figure 12B). These iron-rich minerals contained up to $96 \% \mathrm{FeO}$ and had aluminum contents ranging from 0.1 to $13 \% \mathrm{Al}_{2} \mathrm{O}_{3}$ (table 8 ) and their Raman spectra indicated the presence of magnetite, with characteristic bands at 663 to $652 \mathrm{~cm}^{-1}$ (Faria et al., 1997). However, their atomic proportions, based on stoichiometric calculation as suggested by Droop

Figure 13. A red cubic pyrochlore inclusion displaying halo and radial cracks in darkfield illumination. Photomicrograph by Doan Thi Anh Vu; magnified 50x.

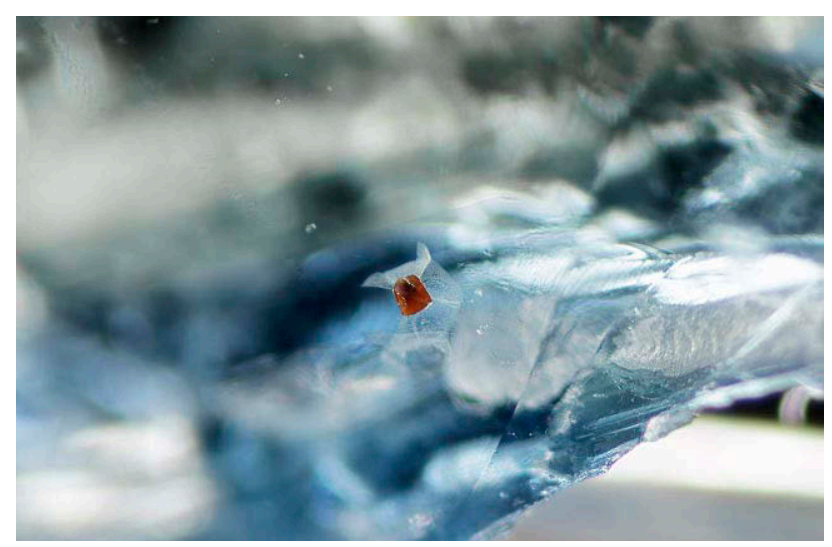

TABLE 7. Representative EPMA analyses of ilmenite inclusions in sapphires from southern Vietnam, Thailand, and Australia.

\begin{tabular}{|c|c|c|c|c|c|}
\hline \multirow{3}{*}{$\begin{array}{l}\text { Mineral } \\
\text { phase } \\
\text { analysis } \\
\text { (wt.\%) }\end{array}$} & \multicolumn{3}{|c|}{ Southern Vietnam } & \multirow{3}{*}{\begin{tabular}{|l} 
Thailand \\
Kanchanaburia
\end{tabular}} & \multirow{3}{*}{$\begin{array}{c}\text { Australia } \\
\text { New } \\
\text { England }\end{array}$} \\
\hline & \multirow{2}{*}{\begin{tabular}{|c|} 
Dak Nong \\
DN39 \\
\end{tabular}} & \multicolumn{2}{|c|}{ Di Linh } & & \\
\hline & & DL23 & DL85 & & \\
\hline $\mathrm{SiO}_{2}$ & 0.11 & 0.10 & nd & 0.00 & 0.70 \\
\hline $\mathrm{TiO}_{2}$ & 34.94 & 37.08 & 33.77 & 45.73 & 59.04 \\
\hline $\mathrm{Al}_{2} \mathrm{O}_{3}$ & 0.02 & 1.50 & 0.58 & 6.90 & 0.45 \\
\hline $\mathrm{Cr}_{2} \mathrm{O}_{3}$ & nd & 0.04 & 0.12 & na & 0.00 \\
\hline $\mathrm{FeO}_{\text {total }}$ & 61.43 & 61.58 & 63.48 & 27.64 & 32.05 \\
\hline $\mathrm{MnO}$ & 0.14 & 0.06 & 0.05 & 17.59 & 0.40 \\
\hline $\mathrm{MgO}$ & nd & 0.18 & 0.64 & 0.98 & 1.85 \\
\hline $\mathrm{ZnO}$ & 0.80 & nd & nd & na & 0.00 \\
\hline $\mathrm{CaO}$ & nd & 0.01 & nd & 0.00 & 0.00 \\
\hline $\mathrm{NiO}$ & 0.02 & nd & 0.03 & na & na \\
\hline Total (oxides) & 97.46 & 100.54 & 98.67 & 98.84 & 94.57 \\
\hline Formula $3(\mathrm{O})$ & & & & & \\
\hline Si & 0.003 & 0.003 & - & 0.000 & 0.017 \\
\hline $\mathrm{Ti}$ & 0.752 & 0.757 & 0.718 & 0.857 & 1.102 \\
\hline $\mathrm{Al}$ & 0.001 & 0.048 & 0.019 & 0.202 & 0.013 \\
\hline $\mathrm{Cr}$ & - & 0.001 & 0.003 & - & 0.000 \\
\hline $\mathrm{Fe}^{3+}$ & 0.655 & 0.584 & 0.715 & 0.124 & 0.000 \\
\hline $\mathrm{Fe}^{2+}$ & 0.814 & 0.814 & 0.786 & 0.452 & 1.330 \\
\hline Mn & 0.003 & 0.001 & 0.001 & 0.371 & 0.008 \\
\hline $\mathrm{Mg}$ & - & 0.007 & 0.027 & 0.036 & 0.068 \\
\hline $\mathrm{Zn}$ & 0.017 & - & - & - & 0.000 \\
\hline $\mathrm{Ca}$ & - & 0.000 & - & 0.000 & 0.000 \\
\hline $\mathrm{Ni}$ & 0.000 & - & 0.001 & - & - \\
\hline Total (cations) & 2.245 & 2.216 & 2.271 & 2.042 & 1.874 \\
\hline$\Sigma \mathrm{R}^{2+}$ & 0.835 & 0.823 & 0.815 & 0.859 & 0.742 \\
\hline$\Sigma \mathrm{R}^{2+}$ & 1.410 & 1.393 & 1.455 & 1.183 & 1.132 \\
\hline Atomic $(\%)$ & & & & & \\
\hline Ilmenite & 53.1 & 54.5 & 49.4 & 56.7 & 98.8 \\
\hline Magnetite & 6.8 & 6.9 & 8.3 & 0.0 & 0.0 \\
\hline Hematite & 38.7 & 33.7 & 39.8 & 29.9 & 0.0 \\
\hline $\begin{array}{l}\text { Abbreviations: } \\
\mathrm{Fe}^{2+} \text { and } \mathrm{Fe}^{3+} \mathrm{W} \\
(1987) \\
\sum \mathrm{R}^{2+}=\mathrm{Fe}^{2+}+\mathrm{Mn} \\
{ }^{2} \text { Khamloet et al } \\
{ }^{\circ} \text { Sutherland et }\end{array}$ & $\begin{array}{l}\text { na }=\text { not an } \\
\text { ere recalcul } \\
+M g+Z n+C \\
\text { (2014) } \\
\text { al. (1998a) }\end{array}$ & $\begin{array}{l}\text { alyzed; nd } \\
\text { lated from } \\
\text { a+Ni; } \Sigma R^{3+}\end{array}$ & $\begin{array}{l}\text { not dete } \\
\text { tal FeO a } \\
=T i+A l+C\end{array}$ & $\begin{array}{l}\text { ed } \\
\text { r the method o } \\
-\mathrm{Fe}^{3+} \text {. }\end{array}$ & of Droop \\
\hline
\end{tabular}

(1987), yielded a $\Sigma \mathrm{R}^{3+} / \Sigma \mathrm{R}^{2+}$ ratio of almost 1:1, particularly $\mathrm{Fe}^{3+} / \mathrm{Fe}^{2+}$ (see table 8 ), which was inconsistent with the formula of magnetite $\left(\mathrm{Fe}_{3} \mathrm{O}_{4}\right)$, with an $\mathrm{Fe}^{3+} / \mathrm{Fe}^{2+}$ ratio of 2:1. More details of these inclusions need to be worked out in the future. 
TABLE 8. Representative EPMA analyses of unidentified iron-rich inclusions in sapphires from southern Vietnam.

\begin{tabular}{|c|c|c|c|c|c|c|c|c|}
\hline \multirow{2}{*}{$\begin{array}{l}\text { Mineral phase } \\
\text { analysis (wt.\%) }\end{array}$} & \multicolumn{2}{|c|}{ Dak Nong } & \multicolumn{2}{|c|}{ Di Linh } & \multicolumn{2}{|c|}{ Krong Nang } & \multicolumn{2}{|c|}{ Binh Thuan } \\
\hline & DN73 & DN89 & DL14 & DL50 & KN05 & KN19 & PT17 & PT18 \\
\hline $\mathrm{SiO}_{2}$ & 0.33 & 0.02 & nd & 0.02 & 1.58 & 0.06 & 0.27 & 0.34 \\
\hline $\mathrm{TiO}_{2}$ & 4.43 & 1.57 & 1.53 & 2.33 & 6.62 & 1.24 & 0.22 & 0.52 \\
\hline $\mathrm{Al}_{2} \mathrm{O}_{3}$ & 12.51 & 8.78 & 6.66 & 0.21 & 10.82 & 11.84 & 0.12 & 10.14 \\
\hline $\mathrm{Cr}_{2} \mathrm{O}_{3}$ & 0.18 & nd & 0.04 & 0.02 & 0.33 & 0.03 & 0.03 & 0.06 \\
\hline $\mathrm{FeO}_{\text {total }}$ & 77.55 & 87.90 & 89.29 & 96.45 & 75.75 & 86.30 & 95.46 & 86.45 \\
\hline $\mathrm{MnO}$ & nd & 0.14 & 1.49 & 0.41 & 0.08 & 0.18 & 1.81 & 1.57 \\
\hline $\mathrm{MgO}$ & 0.98 & 0.18 & 0.29 & 1.36 & 0.15 & 0.37 & 0.88 & 0.05 \\
\hline $\mathrm{ZnO}$ & 0.43 & 0.33 & 0.36 & nd & 0.31 & 0.51 & nd & nd \\
\hline $\mathrm{CaO}$ & 0.12 & nd & nd & nd & 0.12 & nd & nd & nd \\
\hline $\mathrm{NiO}$ & 0.02 & nd & nd & 0.03 & 0.10 & nd & nd & nd \\
\hline Total (oxides) & 96.54 & 98.92 & 99.66 & 100.84 & 95.86 & 100.52 & 98.78 & 99.13 \\
\hline \multicolumn{9}{|l|}{ Formula $32(\mathrm{O})$} \\
\hline $\mathrm{Si}$ & 0.110 & 0.001 & - & 0.008 & 0.523 & 0.020 & 0.104 & 0.116 \\
\hline $\mathrm{Ti}$ & 1.106 & 0.051 & 0.405 & 0.645 & 1.648 & 0.310 & 0.063 & 0.133 \\
\hline $\mathrm{Al}$ & 4.892 & 3.598 & 2.769 & 0.091 & 4.218 & 4.641 & 0.053 & 4.104 \\
\hline $\mathrm{Cr}$ & 0.047 & - & 0.011 & 0.006 & 0.086 & 0.008 & 0.010 & 0.016 \\
\hline $\mathrm{Fe}^{3+}$ & 9.753 & 12.429 & 13.148 & 14.925 & 8.501 & 11.658 & 15.700 & 12.266 \\
\hline $\mathrm{Fe}^{2+}$ & 11.769 & 13.121 & 13.183 & 14.740 & 12.460 & 12.349 & 14.788 & 12.571 \\
\hline$M n$ & - & 0.041 & 0.446 & 0.128 & 0.023 & 0.051 & 0.585 & 0.457 \\
\hline Mg & 0.485 & 0.092 & 0.150 & 0.748 & 0.073 & 0.183 & 0.498 & 0.026 \\
\hline $\mathrm{Zn}$ & 0.105 & 0.085 & 0.095 & - & 0.075 & 0.125 & - & - \\
\hline $\mathrm{Ca}$ & 0.043 & - & - & - & 0.041 & - & - & - \\
\hline $\mathrm{Ni}$ & 0.005 & - & - & 0.008 & 0.027 & - & - & - \\
\hline Total (cations) & 28.315 & 29.784 & 30.206 & 31.299 & 27.676 & 29.345 & 31.801 & 29.690 \\
\hline$\Sigma \mathrm{R}^{2+}$ & 12.407 & 13.339 & 13.874 & 15.624 & 12.699 & 12.708 & 15.871 & 13.054 \\
\hline$\Sigma \mathrm{R}^{3+}$ & 15.908 & 16.078 & 16.333 & 15.667 & 14.453 & 16.617 & 15.826 & 16.519 \\
\hline
\end{tabular}

Pyrochlore was recognized as an inclusion in three sapphire samples from Dak Nong and a couple of samples from Krong Nang. They formed similarly red cubic crystals, which were commonly surrounded by radial cracks (figure 13). Based on SEMEDS analysis, Smith et al. (1995) previously reported uranpyrochlore, $(\mathrm{Ca}, \mathrm{U})_{2}(\mathrm{Ti}, \mathrm{Nb}, \mathrm{Ta})_{2} \mathrm{O}_{6}$, in sapphires from Di Linh and Binh Thuan. EPMA analyses of pyrochlore in this study yielded a high $\mathrm{U}$ content (about $19-22 \%$ in $\Sigma \mathrm{R}^{2+}$, a high $\mathrm{Nb}$ content leading to a $\mathrm{Nb} / \mathrm{Ta}$ ratio $\geq 8$, and $\mathrm{a}(\mathrm{Nb}+\mathrm{Ta}) / \mathrm{Ti}$ ratio of about 2 (table 9), all within the compositional range of uranpyrochlore (Hogarth, 1977). These compositions were similar to those found in Australian sapphires from Anakie (Guo et al., 1996) and New England (Sutherland et al., 1998a).

\section{DISCUSSION}

It has been suggested that sapphire and ruby deposited within basaltic terranes could not have crystallized directly from basaltic magma (Coenraads, 1992; Levinson and Cook, 1994; Guo et al., 1996; Sutherland et al., 1998a, 2002, 2015; Khamloet et al., 2014; Promwongnan and Sutthirat, 2019). A corroded surface is a typical feature observed in basalt-associated sapphires, clearly indicating transport by the hot magma. This has also been reported for sapphires from Di Linh and Binh Thuan (Smith et al., 1995). A petrochemical study of sapphire-associated basalts from Dak Nong has also suggested that these xenocrystic sapphires may have formed in the boundary between the lower crust and upper mantle prior to corrosive transport in alkali basaltic magma 
TABLE 9. Representative EPMA analyses of pyrochlore inclusions found in sapphires from southern Vietnam and Australia.

\begin{tabular}{|c|c|c|c|c|c|c|}
\hline \multirow{3}{*}{$\begin{array}{l}\text { Mineral } \\
\text { phase } \\
\text { analysis } \\
\text { (wt.\%) }\end{array}$} & \multicolumn{4}{|c|}{ Southern Vietnam } & \multicolumn{2}{|c|}{ Australia } \\
\hline & \multicolumn{2}{|c|}{ Dak Nong } & \multicolumn{2}{|c|}{ Krong Nang } & \multirow{2}{*}{ Anakie $^{a}$} & \multirow{2}{*}{$\begin{array}{c}\text { New } \\
\text { England }\end{array}$} \\
\hline & DN35 & DN77 & KN18 & KN36 & & \\
\hline $\mathrm{SiO}_{2}$ & 0.23 & 0.37 & 0.55 & 0.33 & na & 0.00 \\
\hline $\mathrm{TiO}_{2}$ & 11.67 & 11.20 & 10.59 & 11.63 & 11.48 & 10.14 \\
\hline $\mathrm{FeO}$ & 1.68 & 1.38 & 1.54 & 1.57 & 1.51 & 0.30 \\
\hline $\mathrm{MnO}$ & 0.37 & 0.19 & 0.08 & 0.03 & na & na \\
\hline $\mathrm{CaO}$ & 6.86 & 6.63 & 6.26 & 6.89 & 5.58 & 5.49 \\
\hline $\mathrm{Na}_{2} \mathrm{O}$ & 4.33 & 5.11 & 4.44 & 5.24 & 4.27 & 5.92 \\
\hline $\mathrm{K}_{2} \mathrm{O}$ & 0.04 & 0.05 & 0.06 & 0.09 & na & na \\
\hline $\mathrm{Nb}_{2} \mathrm{O}_{5}$ & 33.61 & 32.24 & 33.88 & 33.03 & 32.99 & 38.48 \\
\hline $\mathrm{Ta}_{2} \mathrm{O}_{5}$ & 6.05 & 6.50 & 5.97 & 6.34 & 6.58 & 4.44 \\
\hline $\mathrm{ThO}_{2}$ & 7.88 & 7.31 & 7.00 & 7.61 & 8.56 & 1.99 \\
\hline $\mathrm{UO}_{2}$ & 22.28 & 23.06 & 23.83 & 22.32 & 21.80 & 30.88 \\
\hline $\mathrm{ZrO}_{2}$ & 0.08 & 0.15 & 0.01 & 0.02 & 0.20 & na \\
\hline $\mathrm{Y}_{2} \mathrm{O}_{3}$ & nd & 0.08 & 0.12 & 0.08 & 0.66 & na \\
\hline $\mathrm{Ce}_{2} \mathrm{O}_{3}$ & 0.12 & 0.35 & 0.34 & 0.28 & 0.48 & na \\
\hline $\mathrm{Nd}_{2} \mathrm{O}_{3}$ & 0.14 & 0.17 & nd & 0.06 & 0.54 & na \\
\hline Total (oxides) & 95.36 & 94.40 & 94.67 & 95.51 & 94.90 & 97.64 \\
\hline \multicolumn{7}{|l|}{ Formula $6(\mathrm{O})$} \\
\hline $\mathrm{Si}$ & 0.016 & 0.026 & 0.039 & 0.023 & - & 0.000 \\
\hline $\mathrm{Ti}$ & 0.605 & 0.591 & 0.557 & 0.601 & 0.607 & 0.518 \\
\hline $\mathrm{Fe}$ & 0.097 & 0.081 & 0.090 & 0.090 & 0.089 & 0.017 \\
\hline Mn & 0.022 & 0.011 & 0.005 & 0.002 & - & - \\
\hline $\mathrm{Ca}$ & 0.506 & 0.498 & 0.469 & 0.508 & 0.420 & 0.400 \\
\hline $\mathrm{Na}$ & 0.578 & 0.643 & 0.601 & 0.698 & 0.582 & 0.780 \\
\hline K & 0.003 & 0.004 & 0.005 & 0.008 & - & - \\
\hline $\mathrm{Nb}$ & 1.046 & 1.022 & 1.070 & 1.026 & 1.048 & 1.182 \\
\hline Тa & 0.113 & 0.124 & 0.113 & 0.118 & 0.126 & 0.082 \\
\hline Th & 0.123 & 0.117 & 0.111 & 0.119 & 0.137 & 0.031 \\
\hline U & 0.341 & 0.360 & 0.370 & 0.341 & 0.341 & 0.467 \\
\hline $\mathrm{Zr}$ & 0.003 & 0.005 & 0.000 & 0.001 & 0.007 & 0.000 \\
\hline Y & - & 0.003 & 0.005 & 0.003 & 0.025 & - \\
\hline $\mathrm{Ce}$ & 0.003 & 0.009 & 0.009 & 0.007 & 0.012 & - \\
\hline $\mathrm{Nd}$ & 0.000 & 0.000 & - & 0.000 & 0.014 & - \\
\hline Total (cations) & 3.460 & 3.498 & 3.444 & 3.546 & 3.411 & 3.477 \\
\hline$\Sigma \mathrm{R}^{2+}$ & 1.674 & 1.719 & 1.651 & 1.767 & 1.628 & 1.694 \\
\hline $\mathrm{Nb} / \mathrm{Ta}$ & 9 & 8 & 9 & 9 & 8 & 14 \\
\hline$(\mathrm{Nb}+\mathrm{Ta}) / \mathrm{Ti}$ & 2 & 2 & 2 & 2 & 2 & 2 \\
\hline $\mathrm{U} / \Sigma \mathrm{R}^{2+}$ & 20 & 21 & 22 & 19 & 21 & 28 \\
\hline \multicolumn{7}{|c|}{$\begin{array}{l}\text { Abbreviation: } n a=\text { not analyzed; } n d=\text { not detected } \\
\sum R^{2+}=\mathrm{Fe}+\mathrm{Mn}+\mathrm{Ca}+\mathrm{Na}+\mathrm{K}+\mathrm{Th}+U+\mathrm{Z}+\mathrm{Y}+\mathrm{Ce}+\mathrm{Nd} \\
\text { a Guo et al. (1996) } \\
{ }^{\circ} \text { Sutherland et al. (1998a) }\end{array}$} \\
\hline
\end{tabular}

(Garnier et al., 2005). Moreover, a hypothesis has recently been proposed that the original crystallization of alluvial Dak Nong sapphires would have taken place in the shallow crust within an iron-rich syenite melt in collaboration with carbonate- $\mathrm{H}_{2} \mathrm{O}-\mathrm{CO}_{2}$ fluid phases, based on the geochemistry of syngenetic mineral inclusions (i.e., zircon, columbite, oligoclase, and Al-Ti-hematite) (Izokh et al., 2010).

Based on the results of this detailed study, the most common syngenetic mineral inclusions (ferrocolumbite, alkali feldspar, and zircon) were similar in composition to those in Dak Nong sapphires (Izokh et al., 2010) as well as alkaline felsic inclusion groups in other alluvial basalt-related sapphires (Guo et al., 1996). Although hercynite spinel and ilmenite (titanohematite) inclusions observed in this study were quite different from Al-Ti-hematite in Dak Nong sapphires (containing 85.6 wt. $\% \mathrm{Fe}_{2} \mathrm{O}_{3}, 11.9$ wt. $\% \mathrm{Al}_{2} \mathrm{O}_{3}$, and $1.57 \mathrm{wt} . \% \mathrm{TiO}_{2}$ as reported by Izokh et al., 2010), they still favored igneous sources instead of metamorphic sources. Although columbite and pyrochlore in the studied sapphires were chemically close to the inclusions Guo et al. (1996) proposed as representing a carbonatite assemblage, they were also similar to those found in alluvial basalt-associated sapphires from silicate melt origin (Sutherland et al., 1998a). Therefore, both columbite and pyrochlore may relate to silicate melts to indicate the original source of these sapphires.

Consequently, a unique mineral inclusion suite including alkali feldspar, zircon, hercynitic spinel, and ilmenite appears to have crystallized from alkaline felsic magma, which is relevant to the original source of sapphires from southern Vietnam. This magmatism should have taken place extensively prior to fractionated crystallization in the lower crust. This model agrees well with the genetic model proposed by Aspen et al. (1990), Sutherland et al. (1998a, 2009), Pin et al. (2006), Zaw et al. (2006), Upton et al. (2009), Khamloet et al. (2014), and Promwongnan and Sutthirat (2019).

Based on the geological setting in southern Vietnam, granite and granosyenite occurred in the Triassic and Cretaceous during orogenic periods due to Indosinian-Yangtze (South China) collision and Paleo-Pacific plate subduction, respectively (Carter et al., 2001; Nguyen et al., 2004; Tri and Khuc, 2011; Shellnutt et al., 2013). Subsequently, alkali basaltic magmas in southern Vietnam had probably been derived from garnet peridotite of the asthenosphere at high pressure $(<4 \mathrm{GPa})$ and high temperature (about $1470^{\circ} \mathrm{C}$ ) (Hoang and Flower, 1998), and mixed with recycling oceanic crustal materials from Paleo-Pa- 


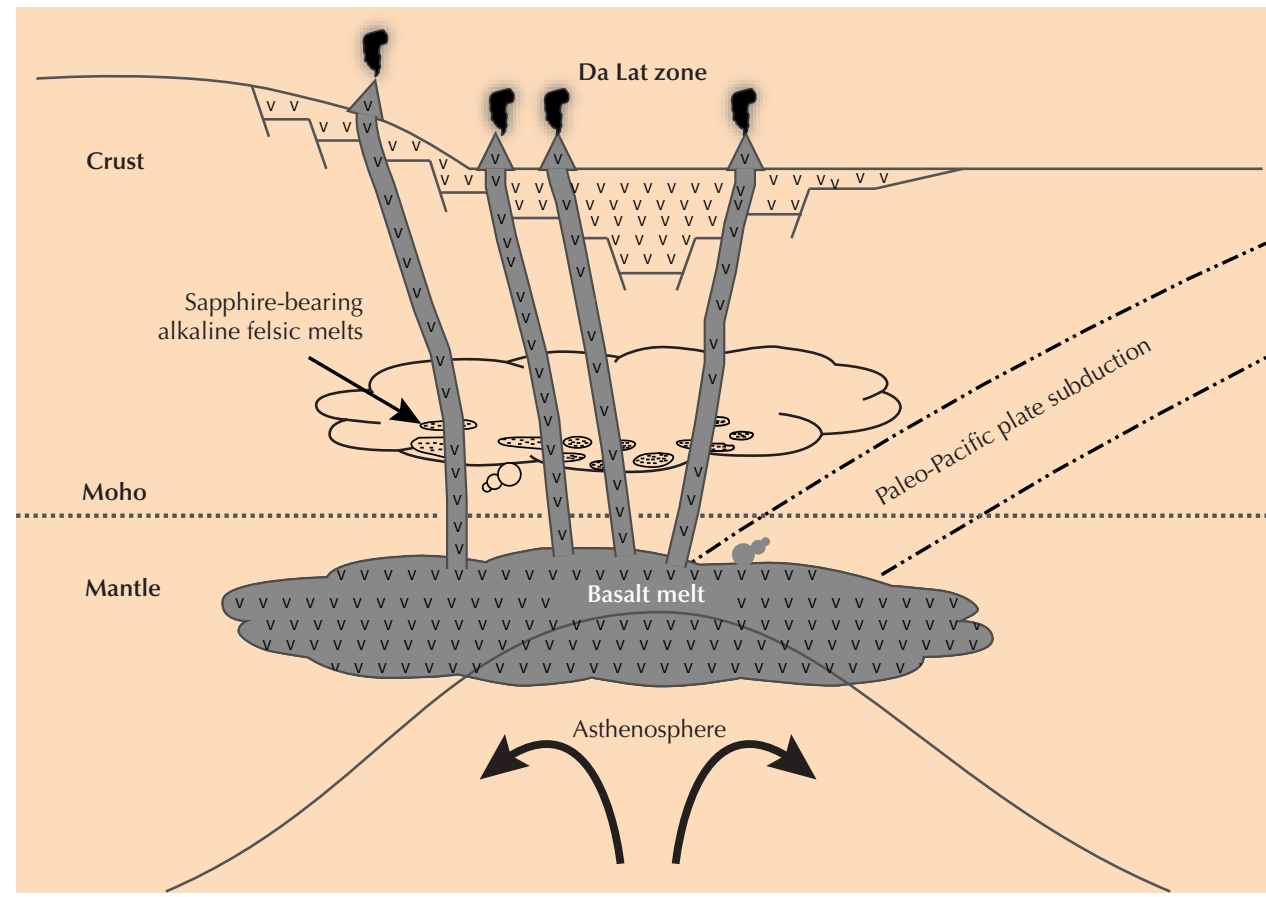

Figure 14. This illustration shows an alkaline felsic melt as the environment for sapphire origin, based on the alkali basalt and crustal evolution models for southern Vietnam (Hoang and Flower, 1998; Tri and Khuc, 2011; Anh et al., 2018).

cific plates that subducted beneath the Southeast Asian continental margin (Anh et al., 2018) during the early Tertiary Indian-Eurasian collision. Rising penetration at close to the Moho, an unconformity zone boundary between lower continental crust and upper mantle, located at about 32-75 km depth (Teng et al., 2013), the heat and volatility separated from these alkaline mafic melts caused the extensive melting of silicate rocks (granite and granosyenite) at lower-crust level with the formation of alkaline felsic melt (figure 14). Sapphires should have crystallized directly during the slow cooling of this alkaline felsic melt. Afterwards, the alkali basaltic magma from the asthenosphere of mantle rose and then brought these sapphires onto the surface via volcanic eruption.

\section{CONCLUSIONS}

Mineral inclusions including ferrocolumbite, zircon, alkali feldspar, hercynite spinel, ilmenite (titanohematite), and pyrochlore were identified in sapphires from the main deposits in southern Vietnam. On the basis of chemical composition, they can be mostly grouped into alkaline felsic suites. An alkaline felsic melt is proposed as the crystallization environment for the original formation of these sapphires. Detailed studies on $\mathrm{U} / \mathrm{Pb}$ dating and trace analysis of zircon inclusions should be carried out to support time scale and original formation of the sapphire hosts. Moreover, unidentified iron-bearing inclusions may give more significant information related to the crystallization environment.

\section{ABOUT THE AUTHORS}

Mrs. Vu is a doctor's degree student in geology at Chulalongkorn University in Bangkok and a lecturer at the Faculty of Geology, University of Science, Vietnam National University in Ho Chi Minh City. Dr. Salam and Dr. Fanka are lecturers at the Geology Department, Faculty of Science, Chulalongkorn University. Dr. Belousova is a research fellow at the Department of Earth and Planetary Science, Macquarie University in Sydney. Dr.Sutthirat (chakkaphan.s@chula.ac.th) is a professor of earth sciences at the Geology Department, Faculty of Science, Chulalongkorn University and an academic advisor to the Gem
Testing Laboratory of the Gem and Jewelry Institute of Thailand (GIT).

\section{ACKNOWLEDGMENTS}

The authors would like to thank all staff members of the Geology Department, Faculty of Science, Chulalongkorn University, for their support in sample preparation and analyses. This research is a part of PhD thesis of the first author who has been supported by the Scholarship Program for ASIAN Countries of Chulalongkorn University. 


\section{REFERENCES}

Anh H.T.H., Choi S.H., Yu Y., Hieu P.T., Hoang N.K., Ryu J. (2018) Geochemical constraints on the spatial distribution of recycled oceanic crust in the mantle source of late Cenozoic basalts, Vietnam. Lithos, Vol. 296, pp. 382-395, http://dx.doi.org/10.1016/j.lithos.2017.11.020

Aspen P., Upton B.G.J., Dicken A.P. (1990) Anorthoclase, sanidine and associated megacrysts in Scottish alkali basalts: High pressure syenitic debris from upper mantle sources? European Journal of Mineralogy, Vol. 2, pp. 503-517, http://dx.doi.org/10.1127/ejm/2/4/0503

Barr S., MacDonald A. (1981) Geochemistry and geochronology of late Cenozoic basalts of Southeast Asia: Summary. Geological Society of America Bulletin, Vol. 92, No. 8, pp. 1069-1142, http://dx.doi.org/10.1130/0016-7606(1981)92\%3C508: GAGOLC\%3E2.0.CO;2

Belousova E., Griffin W., O'Reilly S., Fisher N. (2002) Igneous zircon: Trace element composition as an indicator of source rock type. Contributions to Mineralogy and Petrology, Vol. 143, No. 5, pp. 602-622, http://dx.doi.org/10.1007/s00410-002-0364-7

Carter A., Roques D., Bristow C., Kinny P. (2001) Understanding Mesozoic accretion in Southeast Asia: Significance of Triassic thermotectonism (Indosinian orogeny) in Vietnam. Geology, Vol. 29, No. 3, pp. 211-214, http://dx.doi.org/10.1130/00917613(2001)029

Cassidy K., Groves D.I., Binns R.A. (1988) Manganoan ilmenite formed during regional metamorphism of Archean mafic and ultramafic rocks from Western Australia. Canadian Mineralogist, Vol. 26, pp. 999-1012.

Cerný P., Ercit T.S. (1985) Some recent advances in the mineralogy and geochemistry of $\mathrm{Nb}$ and $\mathrm{Ta}$ in rare-element granitic pegmatites. Bulletin de Minéralogie, Vol. 108, pp. 499-532, http://dx.doi.org/10.3406/bulmi.1985.7846

Coenraads R. (1992) Sapphires and rubies associated with volcanic provinces: Inclusions and surface features shed light on their origin. Australian Gemmologist, Vol. 18, No. 3, pp. 70-78.

Deer W.A., Howie R.A., Zussman J. (2013) An Introduction to the Rock-Forming Minerals. Longman, Essex, UK, 696 pp.

Droop G.T.R. (1987) A general equation for estimating $\mathrm{Fe}^{3+}$ concentrations in ferromagnesian silicates and oxides from microprobe analyses, using stoichiometric criteria. Mineralogical Magazine, Vol. 51, No. 361, pp. 431-435, http://dx.doi.org/10.1180/MINMAG.1987.051.361.10

Faria D.L.A., Silva S.V., Oliveira M.T.M. (1997) Raman microspectroscopy of some iron oxides and oxyhydroxides. Journal of Raman Spectroscopy, Vol. 28, No. 11, pp. 873-878, h t tp: //dx.doi .org / 10 . 1002 /( S I C I ) 1097 4555(199711)28:11\%3C873:AID-JRS177\%3E3.0.CO;2-B

Fuhrman M., Frost B.R., Lindsley D.H. (1988) Crystallization conditions of the Sybille Monzosyenite, Laramie Anorthosite Complex, Wyoming. Journal of Petrology, Vol. 29, No. 3, pp. 699-729, http://dx.doi.org/10.1093/petrology/29.3.699

Garnier V., Ohnenstetter D., Giuliani G., Fallick A.E., Trinh P., Quang V., Van L., Schwarz D. (2005) Basalt petrology, zircon ages and sapphire genesis from Dak Nong, southern Vietnam. Mineralogical Magazine, Vol. 69, No. 1, pp. 21-38, http://dx.doi.org/10.1180/0026461056910233

Guo J., O'Reilly S.Y., Griffin W.L. (1996) Corundum from basaltic terrains: A mineral inclusion approach to the enigma. Contributions to Mineralogy and Petrology, Vol. 122, No. 4, pp. 368386, http://dx.doi.org/10.1007/s004100050134

Hoa T.T., Phuong N.T., Anh T.T., Van V.V., Y N.V., Hoang N., Thanh H.H., Anh P.L., Nien B.A., Hung T.Q., Dung P.T., Lam T.H., Hang H.V., Anh T.V., Chuong V.D., Hung P.V., Quan V.M. and eds. (2005) Study of forming conditions and distribution laws of precious and rare minerals related to magmatic activity in Central Vietnam and Tay Nguyen Highlands. State-level
Project, code DTDL-2003/07, Vol. I, pp. 347 (in Vietnamese).

Hoang N., Flower M. (1998) Petrogenesis of Cenozoic basalts from Vietnam: Implication for origins of a 'diffuse igneous province'. Journal of Petrology, Vol. 39, No. 3, pp. 369-395, http://dx.doi.org/10.1093/petroj/39.3.369

Hoang T.H.A., Choi S.H., Yu Y., Pham T.H., Nguyen K.H., Ryu Y. (2018) Geochemical constraints on the spatial distribution of recycled oceanic crust in the mantle source of late Cenozoic basalts, Vietnam. Lithos, Vol. 296-299, pp. 382-395, http://dx.doi.org/10.1016/j.lithos.2017.11.020

Hogarth D.D. (1977) Classification and nomenclature of the pyrochlore group. American Mineralogist, Vol. 62, No. 5-6, pp. 403-410.

Huong L.T.T., Vuong B.T.S., Khoi N.N., Satitkune S., Wanthanachaisaeng B., Hofmeister W., Häger T., Hauzenberger C. (2016) Geology, gemmological properties and preliminary heat treatment of gem-quality zircon from the Central Highlands of Vietnam. Journal of Gemmology, Vol. 35, No. 4, pp. 308-318, http://dx.doi.org/10.15506/JOG.2016.35.4.308

Hutchison C.S. (1989) Geological Evolution of South-east Asia. Clarendon Press, Oxford, UK.

Izokh A.E., Smirnov S.Z., Egorova V.V., Anh T.T., Kovyazin S.V., Phuong N.T., Kalinina V.V. (2010) The conditions of formation of sapphire and zircon in the areas of alkali-basaltoid volcanism in Central Vietnam. Russian Geology and Geophysics, Vol. 51, No. 7, pp. 719-733, http://dx.doi.org/10.1016/j.rgg.2010.06.001

Khamloet P., Pisutha-Arnond V., Sutthirat, C. (2014) Mineral inclusions in sapphire from the basalt-related deposit in Bo Phloi, Kanchanaburi, western Thailand: Indication of their genesis. Russian Geology and Geophysics, Vol. 55, No. 9, pp. 10871102, http://dx.doi.org/10.1016/j.rgg.2014.08.004

Lee T., Lo C.H., Chung S.L., Chen C.Y., Wang P.L., Lin W.P., Hoang N., Chi C., Yem N. $(1998)^{40} \mathrm{Ar} /{ }^{39} \mathrm{Ar}$ Dating Result of Neogene Basalts in Vietnam and its Tectonic Implication. Mantle Geodynamics and Plate Interactions in East Asia, AGU Monograph, Vol. 27, pp. 317-330, http://dx.doi.org/10.1029/GD027p0317

Lepvrier C., Vuong N.V., Maluski H., Thi P.T., Vu T.V. (2008) Indosinian tectonics in Vietnam. Comptes Rendus Geoscience, Vol. 340, No. 2, pp. 94-111, http://dx.doi.org/10.1016/j.crte.2007.10.005

Levinson A.A., Cook F.A. (1994) Gem corundum in alkali basalt: Origin and occurrence. $G \uplus G$, Vol. 30, No. 4, pp. 253-262, http://dx.doi.org/10.5741/GEMS.30.4.253

Lindsley D.H. (1991) Experimental studies of oxide minerals. In D.H. Lindsley, Ed., Oxide Minerals: Petrologic and Magnetic Significance. Reviews in Mineralogy, Vol. 25, Mineralogical Society of America, pp. 69-106.

Long P.V., Vinh H., Garnier V., Giuliani G., Ohnenstetter D., Lhomme T., Schwarz D., Fallick A.E., Dubessy J., Trinh P.T. (2004) Gem corundum deposits in Vietnam. Journal of Gemmology, Vol. 29, No. 3, pp. 129-147, http://dx.doi.org/10.15506/JOG.2004.29.3.129

Mackay D., Simandl G. (2015) Pyrochlore and columbite-tantalite as indicator minerals for specialty metal deposits. Geochemistry: Exploration, Environment, Analysis, Vol. 15, No. 2-3, pp. 167-178, http://dx.doi.org/10.1144/geochem2014-289

Metcalfe I. (1988) Origin and assembly of South-east Asian continental terranes. Geological Society, London, Special Publications, Vol. 37, No. 1, pp. 101-118, http://dx.doi.org/10.1144/GSL.SP.1988.037.01.08

(1996) Pre-Cretaceous evolution of SE Asian terranes. Geological Society, London, Special Publications, Vol. 106, No. 1, pp.97-122, http://dx.doi.org/10.1144/GSL.SP.1996.106.01.09 - 2009) Late Palaeozoic and Mesozoic tectonic and palaeogeographical evolution of SE Asia. In E. Buffetaut, G. Cuny, J. Le Loeuff, and V. Suteethorn, Eds., Late Palaeozoic and Mesozoic Ecosystems in SE Asia. The Geological Society of London, Special Publication, Vol. 315, No. 1, pp. 7-23. 
(2011) Palaeozoic-Mesozoic history of SE Asia. Geological Society of London Special Publications, Vol. 355, No. 1, pp. $7-$ 35, http://dx.doi.org/10.1144/SP355.2

Nguyen T., Satir M., Siebel W., Chen F. (2004) Granitoids in the Dalat zone, southern Vietnam: Age constraints on magmatism and regional geological implications. International Journal of Earth Sciences, Vol. 93, No. 3, pp. 329-340, http://dx.doi.org/10.1007/s00531-004-0387-6

Palke A.C., Saeseaw S., Renfro N.D., Sun Z., McClure S. (2019) Geographic origin determination of blue sapphire. $G \uplus G$, Vol. 55, No. 4, pp. 536-579, http://dx.doi.org/10.5741/gems.55.4.536

Pin C., Monchoux P., Paquette J.L., Azambre B., Wang P.C., Martin R.F. (2006) Igneous albititic dikes in orogenic lherzolites, Western Pyrenees, France: A possible source for corundum and alkali feldspar xenocrysts in basalt terranes. II. Geochemical and petrogenetic considerations. Canadian Mineralogist, Vol. 44, No. 4, pp. 817-842, http://dx.doi.org/10.2113/gscanmin.44.4.817

Promwongnan S., Sutthirat C. (2019) Mineral inclusions in ruby and sapphire from the Bo Welu gem deposit in Chanthaburi, Thailand. $G \uplus G$, Vol. 55, No. 3, pp. 354-369, http://dx.doi.org/10.5741/GEMS.55.3.354

Rangin C., Huchon P., Le Pichon X., Bellon H., Lepvrier C., Roques D., Hoe N.D., Quynh P.V. (1995) Cenozoic deformation of central and south Vietnam. Tectonophysics, Vol. 251, No. 1-4, pp. 180-196, http://dx.doi.org/10.1016/0040-1951(95)00006-2

Rubatto D., Gebauer D. (2000) Use of cathodoluminescence for U$\mathrm{Pb}$ zircon dating by ion microprobe: Some examples from the Western Alps. In M. Pagel et al., Eds., Cathodoluminescence in Geosciences, Springer, Berlin, pp. 373-400.

Saminpanya S., Sutherland F. (2011) Different origins of Thai area sapphire and ruby, derived from mineral inclusions and co-existing minerals. European Journal of Mineralogy, Vol. 23, No. 4, pp. 683-694, http://dx.doi.org/10.1127/0935-1221/2011/0023-2123

Sengör A.M.C., Altiuner D., Cin A., Ustaömer T., Hsü K.J. (1988) Origin and assembly of the Tethyan orogenic collage at the expense of Gondwana land. In M.G. Audley-Chalerles and A. Hallam, Eds., Gondwana and Tethys. The Geological Society of London, Special Publication, Vol. 37, No. 1, pp. 119-181, http://dx.doi.org/10.1144/GSL.SP.1988.037.01.09

Shellnutt J., Lan C.-Y., Long T., Usuki T., Yang H.-J., Mertzman S., Iizuka Y., Chung S.-L., Wang K.-L., Hsu W.-Y. (2013) Formation of Cretaceous Cordilleran and post-orogenic granites and their microgranular enclaves from the Dalat zone, southern Vietnam: Tectonic implications for the evolution of Southeast Asia. Lithos, Vol. s 182-183, pp. 229-241, http://dx.doi.org/10.1016/j.lithos.2013.09.016

Smith C.P., Kammerling R.C., Keller A.S., Peretti A., Scarratt K.V., Khoa N.D., Repetto S. (1995) Sapphires from southern Vietnam. $G \oplus G$, Vol. 31, No. 3, pp. 168-186, http://dx.doi.org/10.5741/GEMS.31.3.168

Sutherland F., Hoskin P., Fanning C., Coenraads R. (1998a) Models of corundum origin from alkali basaltic terrains: A reappraisal.
Contributions to Mineralogy and Petrology, Vol. 133, No. 4, pp. 356-372, http://dx.doi.org/10.1007/s004100050458

Sutherland F., Schwarz D., Jobbins E.A., Coenraads R., Webb G. (1998b) Distinctive gem corundum suites from discrete basalt fields: A comparative study of Barrington, Australia and West Pailin, Cambodia, Gemfields. Journal of Gemmology, Vol. 26, No. 2, pp. 65-85.

Sutherland F., Bosshart G., Fanning C., Hoskin P.W.O., Coenraads R. (2002) Sapphire crystallization, age and origin, Ban Huai Sai, Laos: Age based on zircon inclusions. Journal of Asian Earth Sciences, Vol. 20, No. 7, pp. 841-849, http://dx.doi.org/10.1016/S13679120(01)00067-0

Sutherland F.L., Zaw K., Meffre S., Giuliani G., Fallick A.E., Graham L., Webb G.B. (2009) Gem-corundum megacrysts from east Australian basalt fields: Trace elements, oxygen isotopes and origins. Australian Journal of Earth Sciences, Vol. 56, No. 7, pp. 1003-1022, http://dx.doi.org/10.1080/08120090903112109

Sutherland F.L., Piilonen P.C., Zaw K., Meffre S., Thompson J. (2015) Sapphire within zircon-rich gem deposits, Bo Loei, Ratanakiri Province, Cambodia: Trace elements, inclusions, U-Pb dating and genesis. Australian Journal of Earth Sciences, Vol. 62, No. 6, pp. 761-773.

Teng J., Zhang Z., Zhang Y., Pi J., Deng Y., Zhang X., Wang C.-Y., Gao R., Liu C. (2013) Moho depth, seismicity and seismogenic structure in China mainland. Tectonophysics, Vol. 627, pp 108-121, http://dx.doi.org/10.1016/j.tecto.2013.11.008

Tri T.V., Khuc V. (2011) Geology and Earth Resources of Vietnam. General Department of Geology and Minerals of Vietnam, Ha Noi, Publishing House for Science and Technology, $634 \mathrm{pp}$.

Upton B.J.G., Finch A.A., Slaby E. (2009) Megacrysts and salic xenoliths in Scottish alkali basalts: Derivatives of deep crustal intrusions and small melt fractions from the upper mantle. Mineralogical Magazine, Vol. 73, No. 6, pp. 943-956, http://dx.doi.org/10.1180/minmag.2009.073.6.943

Vu D.T.A. (2010) Petrographical characteristics and genesis of corundum in Vietnam. University of Science, VNU-Ho Chi Minh City, 120 pp.

- (2018) Gemological characteristics and the quality of sapphire from Krong H'Nang - Dak Lak. Science and Technology Development Journal - Natural Sciences, Vol. 1, No. T5, pp. 263-269, http://dx.doi.org/10.32508/stdjns.v1iT5.559

Williams I.S., Claesson S. (1987) Isotopic evidence for the Precambrian provenance and Caledonian metamorphism of high grade paragneisses from the Seve Nappes, Scandinavian Caledonides. Contributions to Mineralogy and Petrology, Vol. 97, No. 2, pp. 205-217, http://dx.doi.org/10.1007/bf00371240

Zaw K., Sutherland F.L., Dellapasqua F., Ryan C.G., Yui T., Mernagh T.P., Duncan D. (2006) Contrasts in gem corundum characteristics, eastern Australian basaltic fields: Trace elements, fluid/melt inclusions and oxygen isotopes. Mineralogical Magazine Vol. 70, No. 6, pp. 669-687, http://dx.doi.org/10.1180/0026461067060356 